\title{
Schur positivity of skew Schur function differences and applications to ribbons and Schubert classes
}

\author{
Ronald C. King • Trevor A. Welsh • Stephanie \\ J. van Willigenburg
}

Received: 2 April 2007 / Accepted: 10 December 2007 / Published online: 21 December 2007

(C) Springer Science+Business Media, LLC 2007

\begin{abstract}
Some new relations on skew Schur function differences are established both combinatorially using Schützenberger's jeu de taquin, and algebraically using Jacobi-Trudi determinants. These relations lead to the conclusion that certain differences of skew Schur functions are Schur positive. Applying these results to a basis of symmetric functions involving ribbon Schur functions confirms the validity of a Schur positivity conjecture due to McNamara. A further application reveals that certain differences of products of Schubert classes are Schubert positive.
\end{abstract}

Keywords Jacobi-Trudi determinant - Jeu de taquin - Ribbon - Schubert calculus · Schur positive $\cdot$ Skew Schur function $\cdot$ Symmetric function

For Manfred Schocker 1970-2006.

S.J. van Willigenburg was supported in part by the National Sciences and Engineering Research Council of Canada.

R.C. King

School of Mathematics, University of Southampton, University Road, Southampton, Hampshire SO17 1BJ, UK

e-mail: rck@maths.soton.ac.uk

T.A. Welsh

Department of Physics, University of Toronto, 60 St. George Street, Toronto, ON, M5S 1A7 Canada e-mail: taw@maths.soton.ac.uk

S.J. van Willigenburg $(\varangle)$

Department of Mathematics, University of British Columbia, 1984 Mathematics Road, Vancouver, BC, V6T 1Z2 Canada

e-mail: steph@math.ubc.ca 


\section{Introduction}

Recently there has been much research on Schur positivity of differences of skew Schur functions, see for example [3, 8, 12]. In this paper we discover some new Schur positive differences of skew Schur functions and use them to derive a result on the basis of symmetric functions consisting of skew Schur functions indexed by certain ribbons, which is analogous to a well-known result on complete symmetric functions.

This paper is structured as follows. In the remainder of this section we review the necessary background concerning tableaux and skew Schur functions. In Section 2 we derive new relations on differences of skew Schur functions, in particular Lemma 2.2 and Theorem 2.13. Lemma 2.2 is the technical heart of the paper and in view of its importance and their independent interest we offer both a combinatorial and an algebraic proof. The former invokes Schützenberger's jeu de taquin, while the latter is based on Jacobi-Trudi determinants. In Section 3 we apply Theorem 2.13 to obtain various new Schur positive differences of skew Schur functions and resolve a conjecture of McNamara in Theorem 3.3. Finally, in Section 4 we show that the preceding results may be used to establish that certain differences of products of Schubert classes are Schubert positive, in a sense that we define.

\subsection{Partitions and diagrams}

Let $\alpha=\left(\alpha_{1}, \alpha_{2}, \ldots, \alpha_{k}\right)$ be a sequence of integers whose sum, $|\alpha|$, is $N$. If the parts $\alpha_{i}$, of $\alpha$, satisfy $\alpha_{1}, \alpha_{2}, \cdots, \alpha_{k}>0$ then we say that $\alpha$ is a composition of $N$, denoted $\alpha \vDash N$. We also say that $\alpha$ has length $\ell(\alpha):=k$. If $\alpha_{i}=\alpha_{i+1}=\cdots=\alpha_{i+j-1}=a$ we normally denote the subsequence $\alpha_{i}, \alpha_{i+1}, \ldots, \alpha_{i+j-1}$ by $a^{j}$. We denote by () the unique composition of 0 .

For use in some of our proofs later, recall that there exists a bijection between compositions of $N$ and the collection $2^{[N-1]}$ of all subsets of $\{1,2, \ldots, N-1\}$ that sends a composition $\alpha=\left(\alpha_{1}, \ldots, \alpha_{\ell(\alpha)}\right)$ to the set of partial sums $S(\alpha)=\left\{\alpha_{1}\right.$, $\left.\alpha_{1}+\alpha_{2}, \ldots, \alpha_{1}+\alpha_{2}+\cdots+\alpha_{\ell(\alpha)-1}\right\}$. If the parts of the composition $\alpha$ satisfy $\alpha_{1} \geq \alpha_{2} \geq \cdots \geq \alpha_{\ell(\alpha)}$ then we say $\alpha$ is a partition of $N$, denoted $\alpha \vdash N$. For clarity of exposition we usually denote sequences and compositions by $\alpha, \beta, \sigma, \tau$ and partitions by $\lambda, \mu, \nu$, and we will use this convention next.

Three partial orders that exist on partitions $\lambda$ and $\mu$ are

1. the inclusion order: $\mu \subseteq \lambda$ if $\mu_{i} \leq \lambda_{i}$ for all $1 \leq i \leq \ell(\mu)$;

2. the dominance order on partitions $\lambda, \mu \vdash N: \mu \leq_{\text {dom }} \lambda$ if

$$
\mu_{1}+\cdots+\mu_{i} \leq \lambda_{1}+\cdots+\lambda_{i}
$$

for all $i$, where if $i>\ell(\lambda)$ (resp. $i>\ell(\mu))$ then $\lambda_{i}:=0$ (resp. $\mu_{i}:=0$ );

3. the lexicographic order on partitions $\lambda, \mu \vdash N: \mu \leq_{\text {lex }} \lambda$ if for some $i$ we have $\mu_{j}=\lambda_{j}$ for $1 \leq j<i$ and $\mu_{i}<\lambda_{i}$.

It is not hard to see that the lexicographic order extends the dominance order. It is also known, see for example [2], that the cover relations in the dominance order are 
1. $(\lambda, a, b, \mu) \triangleleft_{\text {dom }}(\lambda, a+1, b-1, \mu)$ for $\lambda_{\ell(\lambda)}>a \geq b>\mu_{1}$;

2. $\left(\lambda, a^{n}, \mu\right) \triangleleft_{\text {dom }}\left(\lambda, a+1, a^{n-2}, a-1, \mu\right)$ for $\lambda_{\ell(\lambda)}>a>\mu_{1}$ and $n \geq 3$.

For example, $(3,2) \triangleleft_{d o m}(4,1)$ and $(2,2,2,2) \triangleleft_{\text {dom }}(3,2,2,1)$.

Given a partition $\lambda=\left(\lambda_{1}, \lambda_{2}, \ldots, \lambda_{\ell(\lambda)}\right)$ we can associate with it a (Ferrers or Young) diagram also denoted by $\lambda$ that consists of $\lambda_{i}$ left-justified boxes in row $i$ when read from the top. For ease of referral, boxes will be described by their row and column indices. Furthermore, given two partitions $\lambda, \mu$ such that $\mu \subseteq \lambda$, the skew diagram $\lambda / \mu$ is obtained from the diagram $\lambda$ by removing the subdiagram of boxes $\mu$ from the top left corner. In terms of row and column indices

$$
\lambda / \mu=\{(i, j) \mid(i, j) \in \lambda,(i, j) \notin \mu\} .
$$

For example, if we denote boxes by $\times$ then

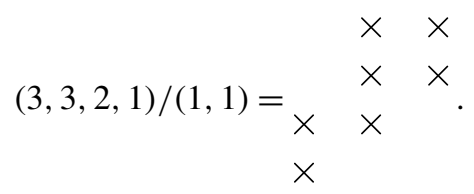

Additionally, two skew diagrams will be considered equivalent if one can be obtained from the other by the removal of empty rows or empty columns. We say a skew diagram is connected if it is edgewise connected.

We can describe skew diagrams $\lambda / \mu$ a third way using 1- and 2-row overlap sequences as defined in [13]. Let $\lambda, \mu$ be diagrams such that $\lambda / \mu$ is a skew diagram occupying $\ell(\lambda)$ rows. Then the 1-row overlap sequence of $\lambda / \mu$ is

$$
r^{(1)}(\lambda / \mu)=\left(\lambda_{1}-\mu_{1}, \lambda_{2}-\mu_{2}, \ldots, \lambda_{\ell(\mu)}-\mu_{\ell(\mu)}, \lambda_{\ell(\mu)+1}, \ldots, \lambda_{\ell(\lambda)}\right)
$$

and the 2-row overlap sequence of $\lambda / \mu$ is

$$
r^{(2)}(\lambda / \mu)=\left(\lambda_{2}-\mu_{1}, \lambda_{3}-\mu_{2}, \ldots, \lambda_{\ell(\mu)+1}-\mu_{\ell(\mu)}, \lambda_{\ell(\mu)+2}, \ldots, \lambda_{\ell(\lambda)}\right) .
$$

Hence, considering our previous skew diagram

$$
r^{(1)}((3,3,2,1) /(1,1))=(2,2,2,1), \quad r^{(2)}((3,3,2,1) /(1,1))=(2,1,1) .
$$

Note that this completely describes $\lambda / \mu$ and so we can also denote the skew diagram by

$$
\lambda / \mu=\left(r^{(1)}(\lambda / \mu) \mid r^{(2)}(\lambda / \mu)\right) .
$$

If $r^{(2)}(\lambda / \mu)=\left(1^{k}\right)$ with $k=\ell(\lambda)-1$ then we say $\lambda / \mu$ is a ribbon (or border strip or rim hook) and since $r^{(2)}(\lambda / \mu)$ is predetermined we can describe the ribbon completely by the composition $r^{(1)}(\lambda / \mu)$.

One last notion that we need for diagrams is that of inner and outer corners. If $\lambda$ is a diagram, then we say $(i, j)$ is an inner corner of $\lambda$ if $(i, j) \in \lambda$ and $\lambda$ with the box in position $(i, j)$ removed is also a diagram. Similarly we say $(i, j)$ is an outer corner of $\lambda$ if $(i, j) \notin \lambda$ and $\lambda$ with a box appended in position $(i, j)$ is also a diagram. For 
example, if $\lambda=(3,3,2,1)$ then the inner corners of $\lambda$ are denoted by $\otimes$ and the outer corners by $\star$ :

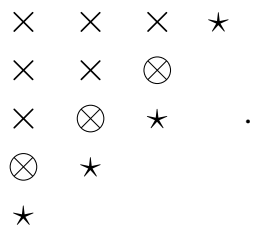

Inner and outer corners will play a vital role in the next subsection.

1.2 Tableaux and jeu de taquin

Consider a skew diagram $\lambda / \mu$. We say we have a tableau, $T$, of shape $\lambda / \mu$ if each box is filled with a positive integer. In addition, we say we have a semistandard Young tableau (SSYT, plural SSYTX) if

1. As we read the entries in each row of $T$ from left to right the entries weakly increase;

2. As we read the entries in each column of $T$ from top to bottom the entries strictly increase.

If we read the entries of $T$ from right to left and top to bottom then the resulting word is called the reading word of $T, w(T)$. If, for each $i$, the number of $i$ s we have read is always at least the number of $(i+1) \mathrm{s}$ we have read then we say $w(T)$ is lattice. Let $c_{i}(T)$ be the total number of $i \mathrm{~s}$ appearing in $T$ and also in $w(T)$, then the list $c(T):=\left(c_{1}(T), c_{2}(T), \ldots\right)$ is known as the content of $T$ and also of $w(T)$. If $T$ is a SSYT with $n$ entries and $c(T)=\left(1^{n}\right)$ then we say $T$ is a standard Young tableau (SYT, plural SYTx).

Lastly, if we have a SSYT we are able to perform Schützenberger's jeu de taquin $(j d t)$, see for example [14], on it.

Definition 1.1 Given a SSYT, $T$, of shape $\lambda / \mu$ we perform a forward-(jdt)-slide as follows:

1. Choose an inner corner of $\mu, C=(i, j)$.

2. Let $c=\min \{T(i+1, j), T(i, j+1)\}$ or $c=T(i+1, j)$ if $T(i+1, j)=T(i, j+1)$ or if only one of $T(i+1, j), T(i, j+1)$ exists then that is taken to be the minimum value. Let $C^{\prime}$ be the position $c$ is in.

3. Form $T^{\prime}$ by setting $T(i, j)=c$ and letting $C^{\prime}$ be empty.

4. Set $C:=C^{\prime}$ and return to the second step until $T^{\prime}$ is a SSYT.

Given a SSYT, $T$, of shape $\lambda / \mu$ we perform a backward-(jdt)-slide as follows:

1. Choose an outer corner of $\lambda, D=(i, j)$.

2. Let $d=\max \{T(i-1, j), T(i, j-1)\} \quad$ or $d=T(i-1, j) \quad$ if $T(i-1, j)=$ $T(i, j-1)$ or if only one of $T(i-1, j), T(i, j-1)$ exists then that is taken to be the maximum value. Let $D^{\prime}$ be the position $d$ is in.

3. Form $T^{\prime \prime}$ by setting $T(i, j)=d$ and letting $D^{\prime}$ be empty. 
4. Set $D:=D^{\prime}$ and return to the second step until $T^{\prime \prime}$ is a SSYT.

Note the output of each algorithm is a SSYT and that these slides are invertible, as illustrated in the following example.

Example 1.2 Let $T={ }_{2} 13^{12}$ and $C=(1,2)$ then a forward-jdt-slide takes place as shown, where $\bullet$ indicates the position of $C$ at each stage.

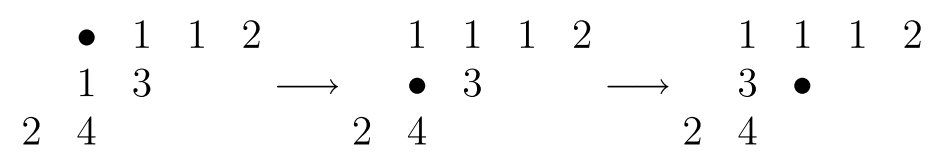

Conversely, let $U=r_{3}^{1} 112$ and $D=(2,3)$ then a backward-jdt-slide takes place as shown, where $\bullet$ indicates the position of $D$ at each stage.

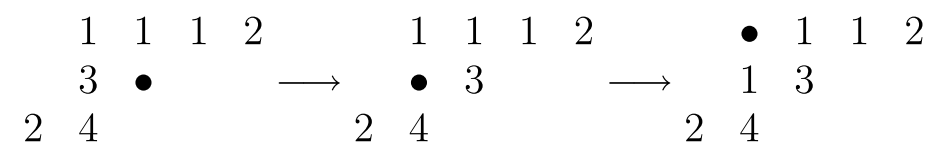

We are now ready to introduce skew Schur functions.

1.3 The algebra of symmetric functions

For any set $\mathcal{T}$ of tableaux, we define the generating function

$$
g(\mathcal{T})=\sum_{T \in \mathcal{T}} x^{c(T)}
$$

where $x^{c(T)}:=x_{1}^{c_{1}(T)} x_{2}^{c_{2}(T)} \ldots$. This generating function can be used to define a basis of the algebra of symmetric functions, $\Lambda$, known as the basis of Schurfunctions $\left\{s_{\lambda}\right\}_{\lambda \vdash N}$ through

$$
s_{\lambda}:=g\left(\mathcal{T}^{\lambda}\right)
$$

where $\mathcal{T}^{\lambda}$ is the set of all SSYTx of shape $\lambda$. Another basis of the algebra of symmetric functions is the basis of complete symmetric functions $\left\{h_{\lambda}\right\}_{\lambda \vdash N}$ where $h_{0}:=1$,

$$
h_{\lambda}:=h_{\lambda_{1}} h_{\lambda_{2}} \cdots h_{\lambda_{\ell(\lambda)}}
$$

and

$$
h_{k}:=\sum_{i_{1} \leq i_{2} \leq \cdots \leq i_{k}} x_{i_{1}} x_{i_{2}} \cdots x_{i_{k}} .
$$

Either of these bases can be used to describe our desired objects of study, skew Schur functions. 
Given a skew diagram $\lambda / \mu$ we define the skew Schur function by

$$
s_{\lambda / \mu}:=g\left(\mathcal{T}^{\lambda / \mu}\right)
$$

where $\mathcal{T}^{\lambda / \mu}$ is the set of all SSYTx of shape $\lambda / \mu$. For clarity of exposition we will use the less conventional overlap notation

$$
s_{\lambda / \mu}=\{\lambda / \mu\}=\left\{r^{(1)}(\lambda / \mu) \mid r^{(2)}(\lambda / \mu)\right\}
$$

to state our results from the next section onwards. In terms of complete symmetric functions

$$
s_{\lambda / \mu}=\left|h_{\lambda_{i}-\mu_{j}-i+j}\right|_{i, j=1}^{\ell(\lambda)}
$$

where $\mu_{j}:=0$ if $j>\ell(\mu)$ and $h_{k}:=0$ if $k<0$, see for example [15]. These determinants are known as Jacobi-Trudi determinants. Expanding, instead, in the Schur function basis we have

$$
s_{\lambda / \mu}=\sum_{\nu} c_{\mu \nu}^{\lambda} s_{\nu}
$$

where $c_{\mu \nu}^{\lambda}$ is the Littlewood-Richardson coefficient defined to be the number of SSYTx, $T$, of shape $\lambda / \mu$ where $w(T)$ is lattice and $c(T)=v$. This manner of determining $c_{\mu \nu}^{\lambda}$ is called the Littlewood-Richardson rule, and clearly yields that $c_{\mu \nu}^{\lambda}$ is a non-negative integer.

In what follows, we are especially interested in the case where $\lambda / \mu$ is a ribbon. In such a case, $\lambda / \mu=\left(\alpha \mid 1^{\ell(\lambda)-1}\right)$ where $\alpha=r^{(1)}(\lambda / \mu)$, and we define $r_{\alpha}=s_{\lambda / \mu} \equiv$ $\left\{\alpha \mid 1^{\ell(\lambda)-1}\right\}$. We call $r_{\alpha}$ a ribbon Schur function. The set $\left\{r_{\lambda}\right\}_{\lambda \vdash N}$ forms another basis for the algebra of symmetric functions [1, Section 2.1]. Ribbon Schur functions are also significant because they are, for example, useful in computing the number of permutations with a given cycle structure and descent set [5], and they can be used to compute skew Schur functions via determinants consisting of associated ribbon Schur functions [7].

Note that the Jacobi-Trudi determinant (1.6) for the ribbon Schur function

$$
r_{\alpha}=\left\{\alpha_{1}, \ldots, \alpha_{\ell(\alpha)} \mid 1^{\ell(\alpha)-1}\right\}
$$

takes the form

$$
r_{\alpha}=\left|h_{R_{i j}}\right|_{i, j=1}^{\ell(\alpha)}
$$

with

$$
R_{i j}= \begin{cases}\alpha_{i}+\alpha_{i+1}+\cdots+\alpha_{j} & \text { if } i \leq j \\ 0 & \text { if } i=j+1 \\ -1 & \text { if } i>j+1\end{cases}
$$


so that

$$
r_{\alpha}=\left|\begin{array}{cccc}
h_{\alpha_{1}} & h_{\alpha_{1}+\alpha_{2}} & h_{\alpha_{1}+\alpha_{2}+\alpha_{3}} & \cdots \\
1 & h_{\alpha_{2}} & h_{\alpha_{2}+\alpha_{3}} & \cdots \\
0 & 1 & h_{\alpha_{3}} & \cdots \\
\vdots & \vdots & \vdots & \ddots
\end{array}\right|
$$

where we have substituted $h_{0}=1$ and $h_{k}=0$ for $k<0$.

More generally, for

$$
s_{\lambda / \mu}=\left\{\alpha_{1}, \ldots, \alpha_{\ell(\alpha)} \mid \beta_{1}+1, \ldots, \beta_{\ell(\alpha)-1}+1\right\}
$$

we have

$$
s_{\lambda / \mu}=\left|h_{Q_{i j}}\right|_{i, j=1}^{\ell(\alpha)}
$$

with

$$
Q_{i j}= \begin{cases}\left(\alpha_{i}+\alpha_{i+1}+\cdots+\alpha_{j}\right)-\left(\beta_{i}+\beta_{i+1}+\cdots+\beta_{j-1}\right) & \text { if } i \leq j-1 \\ \alpha_{i} & \text { if } i=j \\ \beta_{j} & \text { if } i=j+1 \\ -\left(\alpha_{j+1}+\alpha_{j+2}+\cdots+\alpha_{i-1}\right)+\left(\beta_{j}+\beta_{j+1}+\cdots+\beta_{i-1}\right) & \text { if } i>j+1\end{cases}
$$

so that

$$
s_{\lambda / \mu}=\left|\begin{array}{llll}
h_{\alpha_{1}} & h_{\alpha_{1}+\alpha_{2}-\beta_{1}} & h_{\alpha_{1}+\alpha_{2}+\alpha_{3}-\beta_{1}-\beta_{2}} \cdots \\
h_{\beta_{1}} & h_{\alpha_{2}} & h_{\alpha_{2}+\alpha_{3}-\beta_{2}} & \cdots \\
h_{\beta_{1}+\beta_{2}-\alpha_{2}} & h_{\beta_{2}} & h_{\alpha_{3}} & \cdots \\
h_{\beta_{1}+\beta_{2}+\beta_{3}-\alpha_{2}-\alpha_{3}} & h_{\beta_{2}+\beta_{3}-\alpha_{3}} & h_{\beta_{3}} & \\
\vdots & \vdots & \vdots & \cdots
\end{array}\right| .
$$

Note that (1.7) is a non-negative linear combination of Schur functions. This motivates the following definition.

Definition 1.3 If a symmetric function $f \in \Lambda$ can be written as a non-negative linear combination of Schur functions then we say that $f$ is Schur positive.

Our goal for the remainder of this paper is to construct new Schur positive expressions. We end our introduction with a classical Schur positive linear combination that will serve as a motivation for some of our later results.

Theorem 1.4 [9, p. 119] Let $\lambda, \mu \vdash N$ then

$$
h_{\mu}-h_{\lambda}
$$


is Schur positive if and only if $\mu \leq d o m$.

\section{Skew Schur function differences}

In this section we study a variety of differences of skew Schur functions, and moreover discover that some of them are Schur positive. Before we proceed with these differences, we introduce the following hypothesis.

Hypothesis 2.1 Let $\sigma$ and $\tau$ be compositions such that $\ell(\sigma)=s \geq 0$ and $\ell(\tau)=t \geq$ 0 , and let $\bar{\sigma}$ and $\bar{\tau}$ be sequences of non-negative integers that satisfy the following conditions:

1. The lengths of $\bar{\sigma}$ and $\bar{\tau}$ are $s$ and $t$ respectively;

2. $\bar{\sigma}_{s}=1$ when $s>0$;

3. $\bar{\tau}_{1}=1$ when $t>0$;

4. $\bar{\sigma}_{i} \leq \min \left\{\sigma_{i}, \sigma_{i+1}\right\}$ for $1 \leq i<s$, and $\bar{\tau}_{i} \leq \min \left\{\tau_{i}, \tau_{i-1}\right\}$ for $1<i \leq t$.

\subsection{A combinatorial approach}

Lemma 2.2 Assume $\sigma, \tau, \bar{\sigma}, \bar{\tau}$ satisfy Hypothesis 2.1 . If $m \geq 1, n \geq 2$ and $0 \leq x \leq$ $\min \{m, n-1\}$ then

$$
\begin{aligned}
&\{\sigma, m, n, \tau \mid \bar{\sigma}, x, \bar{\tau}\}-\{\sigma, m+1, n-1, \tau \mid \bar{\sigma}, x, \bar{\tau}\} \\
&= \begin{cases}\{\sigma, m, n, \tau \mid \bar{\sigma}, x+1, \bar{\tau}\} & \text { if } x<m ; \\
-\left\{\sigma \backslash \sigma_{s}, m+\sigma_{s}, n, \tau \mid \bar{\sigma} \backslash \bar{\sigma}_{s}, m+1, \bar{\tau}\right\} & \text { if } x=m,\end{cases} \\
&- \begin{cases}\{\sigma, m+1, n-1, \tau \mid \bar{\sigma}, x+1, \bar{\tau}\} & \text { if } x<n-1 ; \\
-\left\{\sigma, m+1, n-1+\tau_{1}, \tau \backslash \tau_{1} \mid \bar{\sigma}, n, \bar{\tau}_{\backslash} \backslash \bar{\tau}_{1}\right\} & \text { if } x=n-1 .\end{cases}
\end{aligned}
$$

If $s=0$ then the term containing $\sigma \backslash \sigma_{s}$ is to be omitted. Similarly, if $t=0$ then the term containing $\tau \backslash \tau_{1}$ is to be omitted.

Proof In this proof, we use Schützenberger's jeu de taquin to construct invertible maps between various sets of tableaux of skew shape, and then use the corresponding generating function (1.2), to obtain (2.1) by means of (1.4) and (1.5). The proof runs through the four cases demarcated by (2.1). First we prove the case where $x<m$ and $x<n-1$. The other three cases are then treated as variants of this case.

Before we begin, we introduce some notation. For any skew diagram $\kappa=(\alpha \mid \beta)$, let $\mathcal{T}^{\kappa}$ denote the set of all SSYTx of shape $\kappa$, so that $\{\kappa\}=g\left(\mathcal{T}^{\kappa}\right)$.

Let

$$
\begin{gathered}
\xi=(\sigma, m, n, \tau \mid \bar{\sigma}, x, \bar{\tau}) \\
\zeta=(\sigma, m+1, n-1, \tau \mid \bar{\sigma}, x, \bar{\tau})
\end{gathered}
$$




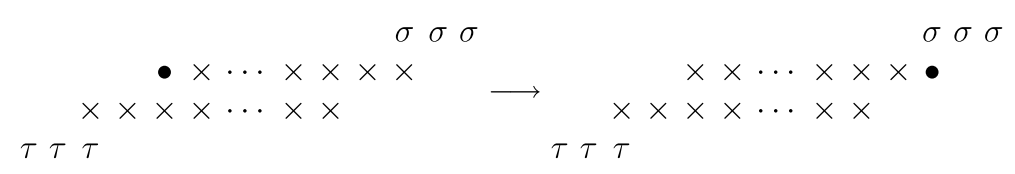

Fig. 1 Map from $\mathcal{T}^{\xi}$ to $\mathcal{U}_{1} \subset \mathcal{T}^{\xi^{*}}$ via a forward-slide

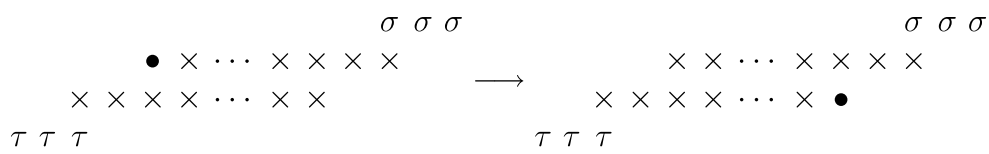

Fig. 2 Map from $\mathcal{T}^{\xi}$ to $\mathcal{U}_{2} \subset \mathcal{T}^{\zeta}$ via a forward-slide

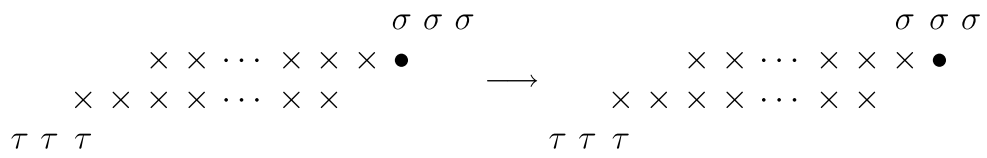

Fig. 3 Map from $\mathcal{U}_{1} \subset \mathcal{T}^{\xi^{*}}$ to $\mathcal{U}_{1}^{<} \subset \mathcal{T}^{\xi^{+}}$via left-shifting the entries $\sigma$

and let $T \in \mathcal{T}^{\xi}$. For the purposes of a forward-jdt-slide, let $C$ be the vacancy immediately to the left of the first entry in the $(s+1)$ th row of $T$.

We now consider our first case, for which $x<m$ and $x<n-1$. The latter of these constraints implies that the vacancy $C$ has just one node from $T$ below it. Thus, forward-sliding $C$ through $T$ necessarily results in this vacancy migrating to the end of either the $(s+1)$ th or $(s+2)$ th row, as depicted in Figs. 1 and 2 respectively.

In these two cases, the resulting SSYT is of shape $\xi^{*}=\left(\sigma, m, n, \tau \mid \bar{\sigma} \backslash \bar{\sigma}_{s}, 0\right.$, $x+1, \bar{\tau})$ or shape $\zeta$ respectively. Let $\mathcal{U}_{1}$ and $\mathcal{U}_{2}$ be the sets of SSYTx of shapes $\xi^{*}$ and $\zeta$ respectively that arise from performing a forward-slide as above on all the elements of $\mathcal{T}^{\xi}$. Then $\{\xi\}=g\left(\mathcal{U}_{1}\right)+g\left(\mathcal{U}_{2}\right)$.

However, neither $\mathcal{U}_{1}$ nor $\mathcal{U}_{2}$ is the full set of SSYTx of shape $\xi^{*}$ or $\zeta$ respectively. In particular, for each tableau $U \in \mathcal{U}_{1}$, the entry at the end of the $(s+1)$ th row (immediately to the left of the vacancy) is larger than that at the beginning of the $s$ th row (immediately above the vacancy), because in the preimage $T$ of $U$, the former of these entries would have been immediately below the latter. We claim that all SSYTx of shape $\xi^{*}$ that satisfy this constraint occur in $\mathcal{U}_{1}$. To see this, let $U$ be an arbitrary such SSYT. Performing a backward-jdt-slide on $U$ necessarily results in a SSYT of shape $\xi$, which is thus an element of $\mathcal{T}^{\xi}$. That forward-sliding is the inverse of backward-sliding then guarantees that $U \in \mathcal{U}_{1}$.

Now form the set $\mathcal{U}_{1}^{<}$of tableaux by, for each tableau $U \in \mathcal{U}_{1}$, shifting each entry in the first $s$ rows of $U$ one position to its left. This shift is indicated in Fig. 3. The elements of $\mathcal{U}_{1}^{<}$are of shape

$$
\xi^{+}=(\sigma, m, n, \tau \mid \bar{\sigma}, x+1, \bar{\tau}) .
$$

The above constraint on the entries of each element of $\mathcal{U}_{1}$ ensures that each element of $\mathcal{U}_{1}^{<}$is a SSYT. Moreover, by reversing the shift, we see that $\mathcal{U}_{1}^{<}=\mathcal{T}^{\xi^{+}}$. Therefore, 


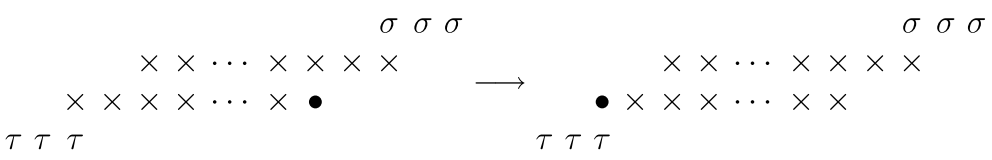

Fig. 4 Map from $\mathcal{T}^{\zeta}$ to $\mathcal{V}_{1} \subset \mathcal{T}^{\zeta^{*}}$ via a backward-slide

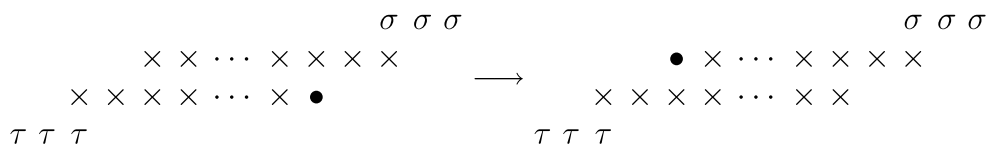

Fig. 5 Map from $\mathcal{T}^{\zeta}$ to $\mathcal{V}_{2} \subset \mathcal{T}^{\xi}$ via a backward-slide

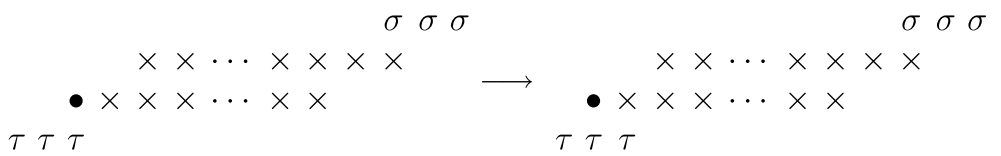

Fig. 6 Map from $\mathcal{V}_{1} \subset \mathcal{T}^{\zeta^{*}}$ to $\mathcal{V}_{1}^{>} \subset \mathcal{T}^{\zeta^{+}}$via right-shifting the entries $\tau$

$\mathcal{T}^{\xi^{+}}$and $\mathcal{U}_{1}$ are in bijection, and thus $\left\{\xi^{+}\right\}=g\left(\mathcal{U}_{1}\right)$. Consequently, $\{\xi\}-\left\{\xi^{+}\right\}=$ $g\left(\mathcal{U}_{2}\right)$.

Now consider $T \in \mathcal{T}^{\zeta}$. For the purposes of a backward-slide, let $D$ be the vacancy immediately to the right of the final entry in the $(s+2)$ th row of $T$. The constraint $x<m$ implies that the vacancy $D$ has just one node from $T$ above it. Thus, backward-sliding $D$ through $T$ necessarily results in this vacancy migrating to the beginning of either the $(s+2)$ th or $(s+1)$ th row. These two instances are depicted in Figs. 4 and 5 respectively. In these two cases, the resulting SSYT is of shape $\zeta^{*}=\left(\sigma, m+1, n-1, \tau \mid \bar{\sigma}, x+1,0, \bar{\tau} \backslash \bar{\tau}_{1}\right)$ or shape $\xi$ respectively. Let $\mathcal{V}_{1}$ and $\mathcal{V}_{2}$ be the sets of SSYTx of shapes $\zeta^{*}$ and $\xi$ respectively that result from performing a backward-slide as above on all the elements of $\mathcal{T}^{\zeta}$. Then $\{\zeta\}=g\left(\mathcal{V}_{1}\right)+g\left(\mathcal{V}_{2}\right)$.

In analogy with the treatment of $\mathcal{U}_{1}$ above, for each element $V \in \mathcal{V}_{1}$ we shift each of the entries in rows $s+3$ and below one position to the right, as indicated in Fig. 6.

This forms a set $\mathcal{V}_{1}^{>}$of SSYTx of shape

$$
\zeta^{+}=(\sigma, m+1, n-1, \tau \mid \bar{\sigma}, x+1, \bar{\tau}) .
$$

This yields $\left\{\zeta^{+}\right\}=g\left(\mathcal{V}_{1}\right)$, and consequently, $\{\zeta\}-\left\{\zeta^{+}\right\}=g\left(\mathcal{V}_{2}\right)$.

Now, backward-sliding maps the elements of $\mathcal{U}_{2} \subset \mathcal{T}^{\zeta}$ bijectively onto a subset of $\mathcal{V}_{2}$ because backward-sliding is inverse to forward-sliding. Similarly, forward-sliding maps the elements of $\mathcal{V}_{2} \subset \mathcal{T}^{\xi}$ bijectively onto a subset of $\mathcal{U}_{2}$ because forward-sliding is inverse to backward-sliding. It follows that $\mathcal{U}_{2}$ and $\mathcal{V}_{2}$ are in bijection, and thus $g\left(\mathcal{U}_{2}\right)=g\left(\mathcal{V}_{2}\right)$. Consequently, $\{\xi\}-\left\{\xi^{+}\right\}=\{\zeta\}-\left\{\zeta^{+}\right\}$, which yields the case of the lemma for which $x<m$ and $x<n-1$.

We now consider the case in which $x<m$ and $x=n-1$. In this case, for $T \in \mathcal{T}^{\xi}$, the vacancy $C$ has more than one entry from $T$ below it (assume for now that $\tau$ is not empty). Then, on performing a forward-slide, the vacancy migrates to one of three 


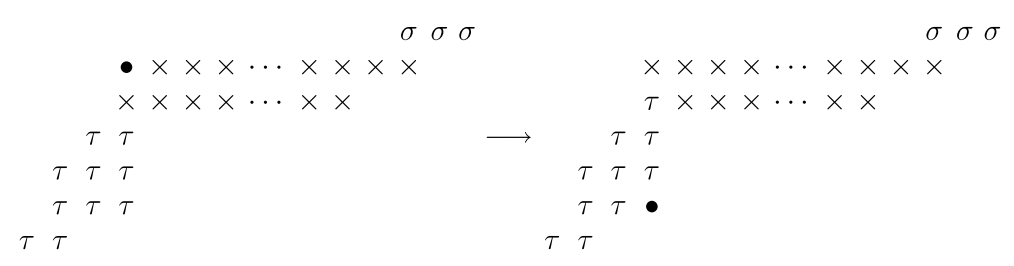

Fig. 7 Map from $\mathcal{T}^{\xi}$ to $\mathcal{U}_{3}$ via a forward-slide

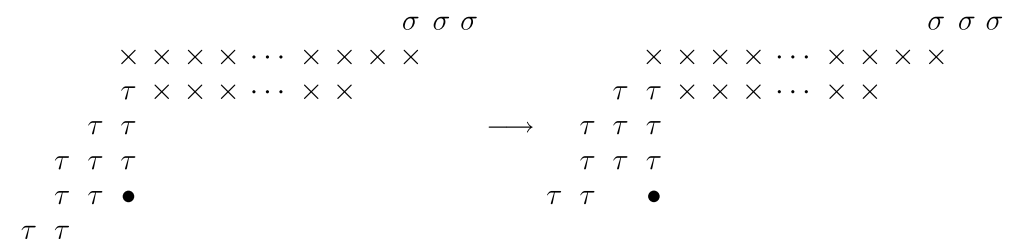

Fig. 8 Map from $\mathcal{U}_{3}$ to $\mathcal{U}_{3}^{\wedge} \subset \mathcal{T}^{\eta}$ via up-shifting of some entries $\tau$

positions. The first two are, as in the first case above, at the ends of rows $s+1$ and $s+2$. The third position is at the bottom of the column that initially contained $C$. This instance is depicted in Fig. 7. Let $\mathcal{U}_{3}$ be the set of all SSYTx that result in this third case. As above, $\mathcal{U}_{1}$ and $\mathcal{U}_{2}$ are defined to be the sets of SSYTx of shapes $\xi^{*}$ and $\zeta$ respectively that result from the first two cases. Then $\{\xi\}=g\left(\mathcal{U}_{1}\right)+g\left(\mathcal{U}_{2}\right)+g\left(\mathcal{U}_{3}\right)$. Moreover, we obtain $\left\{\xi^{+}\right\}=g\left(\mathcal{U}_{1}\right)$ as in the first case, resulting in $\{\xi\}-\left\{\xi^{+}\right\}-$ $g\left(\mathcal{U}_{3}\right)=g\left(\mathcal{U}_{2}\right)$.

Note that, for $U \in \mathcal{U}_{3}$, each entry in the same column of $U$ as the vacancy is greater than or equal to the entry (if there is one) below and to its left. This follows because $U$ was obtained from a SSYT $T \in \mathcal{T}^{\xi}$ by a sequence of slides that move downwards.

Now form the set $\mathcal{U}_{3}^{\wedge}$ by, for each $U \in \mathcal{U}_{3}$, shifting up one position each entry in all the columns to the left of that of the vacancy. This shift is depicted in Fig. 8. Each of the resulting elements of $\mathcal{U}_{3}^{\wedge}$ is of shape

$$
\eta=\left(\sigma, m+1, n-1+\tau_{1}, \tau \backslash \tau_{1} \mid \bar{\sigma}, x+1, \bar{\tau} \backslash \bar{\tau}_{1}\right) .
$$

In view of the above note, each of these elements is a SSYT. Indeed, $\mathcal{U}_{3}^{\wedge}$ is in bijection with $\mathcal{T}^{\eta}$, as may be seen by, in each element of the latter, shifting downward each of the entries in the columns to the left of that of the vacancy and then performing a backward-slide. Therefore, $\{\eta\}=g\left(\mathcal{U}_{3}^{\wedge}\right)=g\left(\mathcal{U}_{3}\right)$. Then, from above, $\{\xi\}-\left\{\xi^{+}\right\}-$ $\{\eta\}=g\left(\mathcal{U}_{2}\right)$. When $\tau$ is empty, the sets $g\left(\mathcal{U}_{3}\right)$ and $g\left(\mathcal{U}_{3}^{\wedge}\right)$ are empty, and the correct expression is given upon setting $\{\eta\}=0$.

Now, as in the first case, consider $T \in \mathcal{T}^{\zeta}$, and for the purposes of a backwardslide, let $D$ be the vacancy immediately to the right of the final entry in the $(s+2)$ th row of $T$. However, $x=n-1$, here, implies that the $(s+1)$ th and $(s+2)$ th rows of $T$ are flush at their left edges, and therefore terms depicted in Fig. 4 do not arise. Consequently, $\{\zeta\}=g\left(\mathcal{V}_{2}\right)$ where, as in the first case, $\mathcal{V}_{2}$ is the set of SSYTx of shape $\xi$ that results from performing a backward-slide on all the elements of $\mathcal{T}^{\zeta}$. 


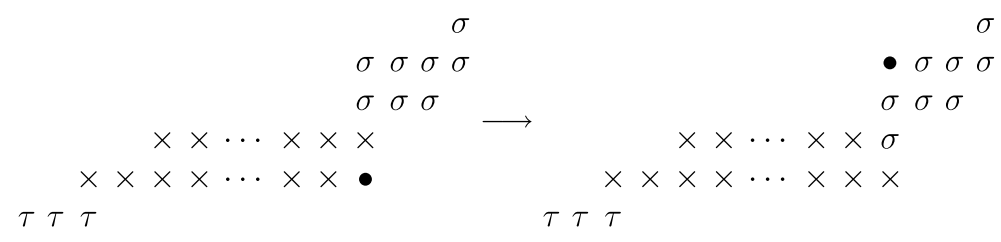

Fig. 9 Map from $\mathcal{T}^{\zeta}$ to $\mathcal{V}_{3}$ via a backward-slide

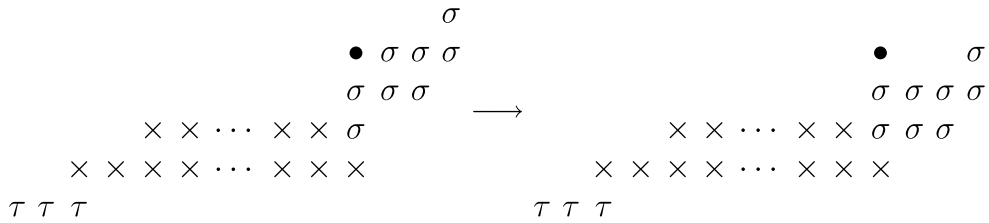

Fig. 10 Map from $\mathcal{V}_{3}$ to $\mathcal{V}_{3}^{\vee} \subset \mathcal{T}^{\gamma}$ via down-shifting of some entries $\sigma$

As in the first case, $\mathcal{U}_{2}$ and $\mathcal{V}_{2}$ are in bijection, whence $g\left(\mathcal{U}_{2}\right)=g\left(\mathcal{V}_{2}\right)$. Thereupon, $\{\xi\}-\left\{\xi^{+}\right\}-\{\eta\}=\{\zeta\}$, and the required expression in the case for which $x<m$ and $x=n-1$ follows.

The case for which $x=m$ and $x<n-1$ is similar to the case just considered, but rotated $180^{\circ}$. Here, rows $(s+1)$ and $(s+2)$ of $T \in \mathcal{T}^{\xi}$ are flush at their right ends. Consequently, forward-sliding $C$ through $T$ necessarily results in this vacancy migrating to the end of the $(s+2)$ th row, as in Fig. 2. The resulting SSYT is then necessarily of shape $\zeta$. With the set $\mathcal{U}_{2}$ formed by enacting this forward-slide on all elements of $\mathcal{T}^{\xi}$, we obtain $\{\xi\}=g\left(\mathcal{U}_{2}\right)$.

Now, as in the previous cases, consider $T \in \mathcal{T}^{\zeta}$, and let $D$ be the vacancy immediately to the right of the final entry in the $(s+2)$ th row of $T$. In this instance, though, $D$ has more than one entry of $T$ above it (provided that $\sigma$ is not empty). Thus in addition to the two terms arising from the migration of $D$ to the beginning of rows $(s+1)$ and $(s+2)$, as in Fig. 4 and Fig. 5 respectively, there is a term arising from the migration of $D$ directly upwards, as in Fig. 9. Let $\mathcal{V}_{3}$ be the set of all SSYTx that result in this third instance. As in the first case, $\mathcal{V}_{1}$ and $\mathcal{V}_{2}$ are defined to be the sets of SSYTx of shapes $\zeta^{*}$ and $\xi$ respectively that result from the first two instances. Then $\{\zeta\}=g\left(\mathcal{V}_{1}\right)+g\left(\mathcal{V}_{2}\right)+g\left(\mathcal{V}_{3}\right)$. Moreover, $\left\{\zeta^{+}\right\}=g\left(\mathcal{V}_{1}\right)$ as in the first case, resulting in $\{\zeta\}-\left\{\zeta^{+}\right\}-g\left(\mathcal{V}_{3}\right)=g\left(\mathcal{V}_{2}\right)$.

The elements of $\mathcal{V}_{3}$ are now treated in analogy with the treatment of $\mathcal{U}_{3}$ above. Namely, we form the set $\mathcal{V}_{3}^{\vee}$ by, for each tableau $V \in \mathcal{V}_{3}$, shifting downward each entry in the columns of $V$ to the right of the vacancy. This shift is depicted in Fig. 10. We see that each element of $\mathcal{V}_{3}^{\vee}$ is a SSYT of shape

$$
\gamma=\left(\sigma \backslash \sigma_{s}, m+\sigma_{s}, n, \tau \mid \bar{\sigma} \backslash \bar{\sigma}_{s}, m+1, \bar{\tau}\right) .
$$

Indeed, by reversing the above construction, we obtain $\mathcal{V}_{3}^{\vee}=\mathcal{T}^{\gamma}$. Therefore, $g\left(\mathcal{V}_{3}^{\vee}\right)=g\left(\mathcal{V}_{3}\right)=\{\gamma\}$. It now follows that $\{\zeta\}-\left\{\zeta^{+}\right\}-\{\gamma\}=g\left(\mathcal{V}_{2}\right)$, where we set $\{\gamma\}=0$ if $\sigma$ is empty. 
As in the previous cases, $\mathcal{U}_{2}$ and $\mathcal{V}_{2}$ are in bijection, whence $g\left(\mathcal{U}_{2}\right)=g\left(\mathcal{V}_{2}\right)$, and thus $\{\xi\}=\{\zeta\}-\left\{\zeta^{+}\right\}-\{\gamma\}$. This yields the required result in the case for which $x=m$ and $x<n-1$.

The case for which $x=m$ and $x=n-1$ is an amalgam of the two previous cases. Here, rows $(s+1)$ and $(s+2)$ of $T \in \mathcal{T}^{\xi}$ are flush at their right ends, but the vacancy $C$ has more than one entry from $T$ below it. Consequently, forward-sliding $C$ through $T$ necessarily results in this vacancy migrating to the end of the $(s+2)$ th row, as in Fig. 2, or to the bottom of the column which originally contained $C$, as in Fig. 7. Then, in comparison with the case for which $x<m$ and $x=n-1$, the set $\mathcal{U}_{1}$ does not arise, and we obtain $\{\xi\}-\{\eta\}=g\left(\mathcal{U}_{2}\right)$. Here again, if $\tau$ is empty, we set $\{\eta\}=0$.

Similarly, the backward-sliding process in this case is as in the case for which $x=m$ and $x<n-1$, except that the set $\mathcal{V}_{1}$ does not arise, and consequently we obtain $\{\zeta\}-\{\gamma\}=g\left(\mathcal{V}_{2}\right)$. Here again, if $\sigma$ is empty, we set $\{\gamma\}=0$.

The familiar bijection between $\mathcal{U}_{2}$ and $\mathcal{V}_{2}$ then implies that $\{\xi\}-\{\eta\}=\{\zeta\}-\{\gamma\}$, giving the required result in this $x=m=n-1$ case, thereby completing the proof of Lemma 2.2.

In order to generalise this result and accommodate special cases of the type appearing in (2.1), it is convenient to introduce the skew Schur functions $K_{\tau, \bar{\tau}}^{\sigma, \bar{\sigma}}(m, n \mid x)$ that, for positive integers $m, n \geq 1,0 \leq x \leq \min \{m, n\}+1$ and $\sigma, \tau, \bar{\sigma}, \bar{\tau}$ satisfying Hypothesis 2.1, are defined as follows:

$$
\begin{aligned}
& K_{\tau, \bar{\tau}}^{\sigma, \bar{\sigma}}(m, n \mid x) \\
& \quad= \begin{cases}\{\sigma, m, n, \tau \mid \bar{\sigma}, x, \bar{\tau}\} & \text { if } x \leq m \text { and } x \leq n ; \\
-\left\{\sigma \backslash \sigma_{s}, m+\sigma_{s}, n, \tau \mid \bar{\sigma} \backslash \bar{\sigma}_{s}, x, \bar{\tau}\right\} & \text { if } x=m+1 \text { and } x \leq n ; \\
-\left\{\sigma, m, n+\tau_{1}, \tau \backslash \tau_{1} \mid \bar{\sigma}, x, \bar{\tau} \backslash \bar{\tau}_{1}\right\} & \text { if } x \leq m \text { and } x=n+1 ; \\
\left\{\sigma \backslash \sigma_{s}, m+\sigma_{s}, n+\tau_{1}, \tau \backslash \tau_{1} \mid \bar{\sigma} \backslash \bar{\sigma}_{s}, x, \bar{\tau} \backslash \bar{\tau}_{1}\right\} & \text { if } x=m+1 \text { and } x=n+1\end{cases}
\end{aligned}
$$

If $s=0$ then the term containing $\sigma \backslash \sigma_{s}$ is to be set to 0 . Similarly, if $t=0$ then the term containing $\tau \backslash \tau_{1}$ is to be set to 0 .

Lemma 2.3 Let $m, n, m^{\prime}, n^{\prime}$ be positive integers for which $m+n=m^{\prime}+n^{\prime}$ and let $x, x^{\prime}$ be non-negative integers for which $x, x^{\prime} \leq \min \left\{m, n, m^{\prime}, n^{\prime}\right\}+1$ then

$$
K_{\tau, \bar{\tau}}^{\sigma, \bar{\sigma}}(m, n \mid x)-K_{\tau, \bar{\tau}}^{\sigma, \bar{\sigma}}\left(m^{\prime}, n^{\prime} \mid x\right)=K_{\tau, \bar{\tau}}^{\sigma, \bar{\sigma}}\left(m, n \mid x^{\prime}\right)-K_{\tau, \bar{\tau}}^{\sigma, \bar{\sigma}}\left(m^{\prime}, n^{\prime} \mid x^{\prime}\right) .
$$

Proof We first write the equation (2.1) in the form

$$
\begin{aligned}
& K_{\tau, \bar{\tau}}^{\sigma, \bar{\sigma}}(p, q \mid y)-K_{\tau, \bar{\tau}}^{\sigma, \bar{\sigma}}(p+1, q-1 \mid y) \\
& \quad=K_{\tau, \bar{\tau}}^{\sigma, \bar{\sigma}}(p, q \mid y+1)-K_{\tau, \bar{\tau}}^{\sigma, \bar{\sigma}}(p+1, q-1 \mid y+1)
\end{aligned}
$$

where $p \geq 1, q \geq 2$, and $0 \leq y \leq \min \{p, q-1\}$. If, in addition, $y<y^{\prime} \leq \min \{p+$ $1, q\}$, then repeated use of (2.4) yields

$$
K_{\tau, \bar{\tau}}^{\sigma, \bar{\sigma}}(p, q \mid y)-K_{\tau, \bar{\tau}}^{\sigma, \bar{\sigma}}(p+1, q-1 \mid y)
$$




$$
=K_{\tau, \bar{\tau}}^{\sigma, \bar{\sigma}}\left(p, q \mid y^{\prime}\right)-K_{\tau, \bar{\tau}}^{\sigma, \bar{\sigma}}\left(p+1, q-1 \mid y^{\prime}\right) .
$$

Without loss of generality, we only need to prove (2.3) in the case for which $m<$ $m^{\prime}$ and $x<x^{\prime}$. In this case

$$
\begin{aligned}
K_{\tau, \bar{\tau}}^{\sigma, \bar{\sigma}}(m, n \mid x)-K_{\tau, \bar{\tau}}^{\sigma, \bar{\sigma}}\left(m^{\prime}, n^{\prime} \mid x\right) \\
=\sum_{i=m}^{m^{\prime}-1}\left(K_{\tau, \bar{\tau}}^{\sigma, \bar{\sigma}}(i, m+n-i \mid x)-K_{\tau, \bar{\tau}}^{\sigma, \bar{\sigma}}(i+1, m+n-i-1 \mid x)\right) \\
=\sum_{i=m}^{m^{\prime}-1}\left(K_{\tau, \bar{\tau}}^{\sigma, \bar{\sigma}}\left(i, m+n-i \mid x^{\prime}\right)-K_{\tau, \bar{\tau}}^{\sigma, \bar{\sigma}}\left(i+1, m+n-i-1 \mid x^{\prime}\right)\right) \\
=K_{\tau, \bar{\tau}}^{\sigma, \bar{\sigma}}\left(m, n \mid x^{\prime}\right)-K_{\tau, \bar{\tau}}^{\sigma, \bar{\sigma}}\left(m^{\prime}, n^{\prime} \mid x^{\prime}\right)
\end{aligned}
$$

where (2.5) has been used to convert each summand of the first sum into the corresponding summand of the second sum. This proves (2.3).

\subsection{An algebraic approach}

As an alternative to the above combinatorial approach to Lemmas 2.2 and 2.3, an algebraic approach may be based on the use of the Jacobi-Trudi determinants, (1.6) or (1.13), which express a skew Schur function in terms of complete symmetric functions.

First, it is convenient to introduce the following family of determinants:

$$
\begin{aligned}
& H_{T}^{S}(m, n \mid z)= \\
& \left|\begin{array}{llllllll}
h_{S_{1,1}} & \cdots & h_{S_{1, s}} & h_{S_{1, s}+m} & h_{S_{1, s}+m+n-z} & h_{S_{1, s}+m+n-z+T_{1,1}} & \cdots & h_{S_{1, s}+m+n-z+T_{1, t}} \\
\vdots & \ddots & \vdots & \vdots & \vdots & \vdots & \ddots \\
h_{S_{s, 1}} & \cdots & h_{S_{s, s}} & h_{S_{s, s}+m} & h_{S_{s, s}+m+n-z} & h_{S_{s, s}+m+n-z+T_{1,1}} & \cdots & h_{S_{s, s}+m+n-z+T_{1, t}} \\
0 & \cdots & 1 & h_{m} & h_{m+n-z} & h_{m+n-z+T_{1,1}} & \cdots & h_{m+n-z+T_{1, t}} \\
0 & \cdots & 0 & h_{z} & h_{n} & h_{n+T_{1,1}} & \cdots & h_{n+T_{1, t}} \\
0 & \cdots & 0 & 0 & 1 & h_{T_{1,1}} & \cdots & h_{T_{1, t}} \\
\vdots & \ddots & \vdots & \vdots & \vdots & \ddots & \vdots \\
0 & \cdots & 0 & 0 & 0 & h_{T_{t, 1}} & \cdots & h_{T_{t, t}}
\end{array}\right|
\end{aligned}
$$

where $S$ and $T$ are $s \times s$ and $t \times t$ matrices of integers, with $s$ and $t$ non-negative integers, while $m$ and $n$ are positive integers and $z$ is any integer. As usual $h_{k}=0$ for $k<0$ and $h_{0}=1$. It is to be understood that any sequence $0 \cdots 1$, whether vertical or horizontal, is a sequence of $0 \mathrm{~s}$ followed by a single 1 in the position indicated.

With this definition we have the following:

Lemma 2.4 Let $m, n, m^{\prime}, n^{\prime}$ be positive integers such that $m+n=m^{\prime}+n^{\prime}$. Then the difference

$$
H_{T}^{S}(m, n \mid z)-H_{T}^{S}\left(m^{\prime}, n^{\prime} \mid z\right)
$$


is independent of $z$ for all integers $z$.

Proof Since $H_{T}^{S}(m, n \mid z)$ is the determinant of a matrix that is block triangular, save for the single subdiagonal element $h_{z}$, it may be evaluated by adding to the determinant of the block triangular matrix the contribution arising from $h_{z}$ and its cofactor. This follows from the fact that the expansion of the determinant $H_{T}^{S}(m, n \mid z)$ is linear in $h_{z}$, that is to say of the form $X+h_{z} Y$, with the term $X$ calculated by setting $h_{z}=0$ and the term $Y$ equal to the cofactor of $h_{z}$. Since, in addition, the determinant of a block triangular matrix is the product of the determinant of its diagonal blocks, we have

$$
\begin{aligned}
& H_{T}^{S}(m, n \mid z)= \\
& \left|\begin{array}{llll}
h_{S_{1,1}} & \cdots & h_{S_{1, s}} & h_{S_{1, s}+m} \\
\vdots & \ddots & \vdots & \vdots \\
h_{S_{s, 1}} & \cdots & h_{S_{s, s}} & h_{S_{s, s}+m} \\
0 & \cdots & 1 & h_{m}
\end{array}\right| \cdot\left|\begin{array}{llll}
h_{n} & h_{n+T_{1,1}} & \cdots & h_{n+T_{1, t}} \\
1 & h_{T_{1,1}} & \cdots & h_{T_{1, t}} \\
\vdots & \vdots & \ddots & \vdots \\
0 & h_{T_{t, 1}} & \cdots & h_{T_{t, t}}
\end{array}\right|
\end{aligned}
$$

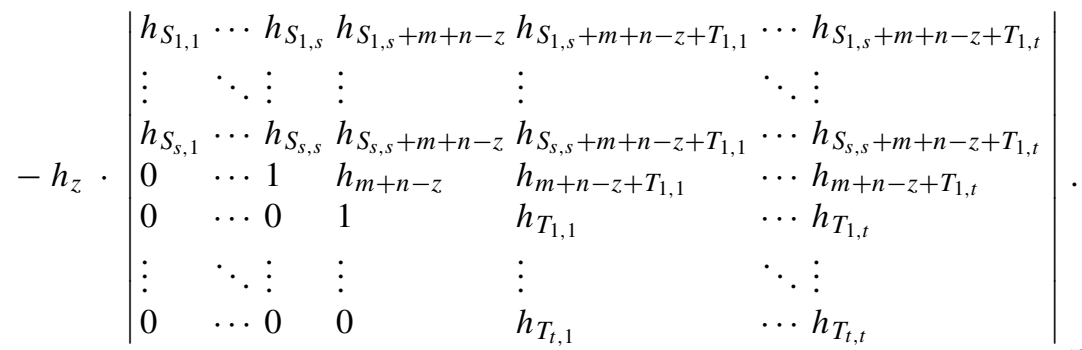

It can be seen from this that for all $m, n, m^{\prime}, n^{\prime} \in \mathbb{N}$ with $m+n=m^{\prime}+n^{\prime}$ and for all integers $z$ we have the identity

$$
\begin{aligned}
& H_{T}^{S}(m, n \mid z)-H_{T}^{S}\left(m^{\prime}, n^{\prime} \mid z\right) \\
& =\left|\begin{array}{llll}
h_{S_{1,1}} & \cdots & h_{S_{1, s}} & h_{S_{1, s}+m} \\
\vdots & \ddots & \vdots & \vdots \\
h_{S_{s, 1}} & \cdots & h_{S_{s, s}} & h_{S_{s, s}+m} \\
0 & \cdots & 1 & h_{m}
\end{array}\right| \cdot\left|\begin{array}{llll}
h_{n} & h_{n+T_{1,1}} & \cdots & h_{n+T_{1, t}} \\
1 & h_{T_{1,1}} & \cdots & h_{T_{1, t}} \\
\vdots & \vdots & \ddots & \vdots \\
0 & h_{T_{t, 1}} & \cdots & h_{T_{t, t}}
\end{array}\right| \\
& -\left|\begin{array}{llll}
h_{S_{1,1}} & \cdots & h_{S_{1, s}} & h_{S_{1, s}+m^{\prime}} \\
\vdots & \ddots & \vdots & \vdots \\
h_{S_{s, 1}} & \cdots & h_{S_{S, s}} & h_{S_{s, s}+m^{\prime}} \\
0 & \cdots & 1 & h_{m^{\prime}}
\end{array}\right| \cdot\left|\begin{array}{llll}
h_{n^{\prime}} & h_{n^{\prime}+T_{1,1}} & \cdots & h_{n^{\prime}+T_{1, t}} \\
1 & h_{T_{1,1}} & \cdots & h_{T_{1, t}} \\
\vdots & \vdots & \ddots & \vdots \\
0 & h_{T_{t, 1}} & \cdots & h_{T_{t, t}}
\end{array}\right|
\end{aligned}
$$

where it is to be noted that the resulting expression is independent of $z$, as claimed.

To make the connection with Lemmas 2.2 and 2.3 by means of Jacobi-Trudi determinants, we introduce the following hypothesis. 
Hypothesis 2.5 Let $\sigma, \tau, \bar{\sigma}, \bar{\tau}$ satisfy Hypothesis 2.1, and let the matrices $S$ and $T$ have matrix elements defined in terms of these by

$$
S_{i j}= \begin{cases}\left(\sigma_{i}+\sigma_{i+1}+\cdots+\sigma_{j}\right) & \\ \quad-\left(\bar{\sigma}_{i}+\bar{\sigma}_{i+1}+\cdots+\bar{\sigma}_{j-1}\right)-i+j & \text { if } i \leq j-1 \\ \sigma_{i} & \text { if } i=j \\ \bar{\sigma}_{j}-1 & \text { if } i=j+1 \\ -\left(\sigma_{j+1}+\sigma_{j+2}+\cdots+\sigma_{i-1}\right) & \text { if } i>j+1 \\ \quad+\left(\bar{\sigma}_{j}+\bar{\sigma}_{j+1}+\cdots+\bar{\sigma}_{i-1}\right)-i+j\end{cases}
$$

for $1 \leq i, j \leq s$, and

$$
T_{i j}= \begin{cases}\left(\tau_{i}+\tau_{i+1}+\cdots+\tau_{j}\right) & \\ \quad-\left(\bar{\tau}_{i}+\bar{\tau}_{i+1}+\cdots+\bar{\tau}_{j-1}\right)-i+j & \text { if } i \leq j-1 \\ \tau_{i} & \text { if } i=j \\ \bar{\tau}_{j}-1 & \text { if } i=j+1 \\ -\left(\tau_{j+1}+\tau_{j+2}+\cdots+\tau_{i-1}\right) & \\ \quad+\left(\bar{\tau}_{j}+\bar{\tau}_{j+1}+\cdots+\bar{\tau}_{i-1}\right)-i+j & \text { if } i>j+1\end{cases}
$$

for $1 \leq i, j \leq t$.

We then have

Lemma 2.6 Assume that $S$ and $T$ satisfy Hypothesis 2.5. If $0 \leq x \leq \min \{m, n\}+1$ then

$$
H_{T}^{S}(m, n \mid x-1)=K_{\tau, \bar{\tau}}^{\sigma, \bar{\sigma}}(m, n \mid x)
$$

Proof Let $z=x-1$. In the case $z<\min \{m, n\}$, under the given hypothesis regarding $S$ and $T$, comparing the determinant (2.7) with the general Jacobi-Trudi determinant (1.13) for a skew Schur function, expressed in overlap notation by way of (1.12), and noting that $\sigma_{s}=\tau_{1}=1$, immediately gives

$$
H_{T}^{S}(m, n \mid x-1)=\{\sigma, m, n, \tau \mid \bar{\sigma}, x, \bar{\tau}\}
$$

Thanks to the definition (2.2), this proves (2.13) in the case $x \leq \min \{m, n\}$.

In the $z=m<n$ case, if $s=0$ then $H_{T}^{S}(m, n \mid m)=0$ since the first and second rows of determinant (2.7) coincide. On the other hand, for $s>0$ the entries of $H_{T}^{S}(m, n \mid m)$ in the $(s+1)$ th and $(s+2)$ th rows all coincide apart from a single entry 1 in the $(s+1)$ th row. Consideration of the cofactor of this element then yields

$$
H_{T}^{S}(m, n \mid m)=-\left\{\sigma \backslash \sigma_{s}, m+\sigma_{s}, n, \tau \mid \bar{\sigma} \backslash \bar{\sigma}_{s}, m+1, \bar{\tau}\right\}
$$

for $s>0$. Once again, the definition (2.2) is such that this proves (2.13) in the case $x-1=m<n$.

Similarly in the $z=n<m$ case, if $t=0$ then $H_{T}^{S}(m, n \mid n)=0$ since the $(s+1)$ th and $(s+2)$ th columns coincide. For $t>0$ the entries of $H_{T}^{S}(m, n \mid n)$ in the $(s+1)$ th 
and $(s+2)$ th columns coincide apart from a single entry 1 in the $(s+2)$ th column. This time, consideration of the cofactor of this element yields

$$
H_{T}^{S}(m, n \mid n)=-\left\{\sigma, m, n+\tau_{1}, \tau \backslash \tau_{1} \mid \bar{\sigma}, n+1, \bar{\tau} \backslash \bar{\tau}_{1}\right\}
$$

for $t>0$. Via the definition (2.2), this proves (2.13) in the case $x-1=n<m$.

Finally, the case $z=m=n$ is such that $H_{T}^{S}(m, m \mid m)=0$ if either $s=0$ or $t=$ 0 since the determinant involves two identical rows or columns, respectively. For $s, t>0$ the determinant involves two rows identical save for a single entry 1 and two columns identical save for another single entry 1 . Subtracting rows, taking the cofactor of the 1 , and then doing the same for the columns gives

$$
H_{T}^{S}(m, m \mid m)=\left\{\sigma \backslash \sigma_{s}, m+\sigma_{s}, m+\tau_{1}, \tau \backslash \tau_{1} \mid \bar{\sigma} \backslash \bar{\sigma}_{s}, m+1, \bar{\tau} \backslash \bar{\tau}_{1}\right\}
$$

for $s>0$ and $t>0$. Again via (2.2), this proves (2.13) in the final $x-1=n=m$ case.

This identification of $H_{T}^{S}(m, n \mid x-1)$, for $S$ and $T$ satisfying Hypothesis 2.5, offers us an alternative proof of Lemma 2.2.

Alternative proof of Lemma 2.2 For all $s \times s$ and $t \times t$ matrices $S$ and $T$, it follows from Lemma 2.4 that for all $m \geq 1, n \geq 2$ and all integers $x$ we have

$H_{T}^{S}(m, n \mid x-1)-H_{T}^{S}(m+1, n-1 \mid x-1)=H_{T}^{S}(m, n \mid x)-H_{T}^{S}(m+1, n-1 \mid x)$.

If we then assume that $S$ and $T$ satisfy Hypothesis 2.5 , and restrict $x$ so that $0 \leq x \leq$ $\min \{m, n-1\}$, we can apply Lemma 2.6 to each term. This gives

$$
\begin{aligned}
& K_{\tau, \bar{\tau}}^{\sigma, \bar{\sigma}}(m, n \mid x)-K_{\tau, \bar{\tau}}^{\sigma, \bar{\sigma}}(m+1, n-1 \mid x) \\
& \quad=K_{\tau, \bar{\tau}}^{\sigma, \bar{\sigma}}(m, n \mid x+1)-K_{\tau, \bar{\tau}}^{\sigma, \bar{\sigma}}(m+1, n-1 \mid x+1) .
\end{aligned}
$$

However, thanks to the definition (2.2), this is just (2.1).

In fact, it is no harder to prove Lemma 2.3.

Alternative proof of Lemma 2.3 Once again for all $s \times s$ and $t \times t$ matrices $S$ and $T$, it follows from Lemma 2.4 that for all positive integers $m, n, m^{\prime}, n^{\prime}$ such that $m+n=$ $m^{\prime}+n^{\prime}$, and all integers $x, x^{\prime}$ we have

$$
H_{T}^{S}(m, n \mid x-1)-H_{T}^{S}\left(m^{\prime}, n^{\prime} \mid x-1\right)=H_{T}^{S}\left(m, n \mid x^{\prime}-1\right)-H_{T}^{S}\left(m^{\prime}, n^{\prime} \mid x^{\prime}-1\right) .
$$

Then assuming that $S$ and $T$ satisfy Hypothesis 2.5, and restricting $x$ and $x^{\prime}$ so that $0 \leq x, x^{\prime} \leq \min \left\{m, n, m^{\prime}, n^{\prime}\right\}+1$, we can simply apply Lemma 2.6 to each term, giving

$$
K_{\tau, \bar{\tau}}^{\sigma, \bar{\sigma}}(m, n \mid x)-K_{\tau, \bar{\tau}}^{\sigma, \bar{\sigma}}\left(m^{\prime}, n^{\prime} \mid x\right)=K_{\tau, \bar{\tau}}^{\sigma, \bar{\sigma}}\left(m, n \mid x^{\prime}\right)-K_{\tau, \bar{\tau}}^{\sigma, \bar{\sigma}}\left(m^{\prime}, n^{\prime} \mid x^{\prime}\right)
$$

as required. 
2.3 Simple skew Schur function differences

Now we derive four straightforward corollaries of Lemma 2.3.

Corollary 2.7 Assume $\sigma, \tau, \bar{\sigma}, \bar{\tau}$ satisfy Hypothesis 2.1. If $0 \leq x \leq m$ then

$$
\begin{aligned}
\{\sigma, m, m+1, \tau \mid \bar{\sigma}, x, \bar{\tau}\}-\{\sigma, m+1, m, \tau \mid \bar{\sigma}, x, \bar{\tau}\} \\
=\quad-\left\{\sigma \backslash \sigma_{s}, m+\sigma_{s}, m+1, \tau \mid \bar{\sigma} \backslash \bar{\sigma}_{s}, m+1, \bar{\tau}\right\} \\
+\left\{\sigma, m+1, m+\tau_{1}, \tau \backslash \tau_{1} \mid \bar{\sigma}, m+1, \bar{\tau} \backslash \bar{\tau}_{1}\right\} .
\end{aligned}
$$

If $s=0$ then omit the first term. Similarly, if $t=0$ then omit the second term.

Proof This is the special case

$$
\begin{aligned}
& K_{\tau, \bar{\tau}}^{\sigma, \bar{\sigma}}(m, m+1 \mid x)-K_{\tau, \bar{\tau}}^{\sigma, \bar{\sigma}}(m+1, m \mid x) \\
& \quad=K_{\tau, \bar{\tau}}^{\sigma, \bar{\sigma}}(m, m+1 \mid m+1)-K_{\tau, \bar{\tau}}^{\sigma, \bar{\sigma}}(m+1, m \mid m+1)
\end{aligned}
$$

of Lemma 2.3, rewritten using (2.2). Note that the terms on the left are each the first case of (2.2), and the terms on the right are the second and third cases, respectively.

The next corollary was proved independently by McNamara [10].

Corollary 2.8 Assume $\sigma, \tau, \bar{\sigma}, \bar{\tau}$ satisfy Hypothesis 2.1 . If $0 \leq x<n \leq m$ then

$$
\begin{aligned}
\{\sigma, m, n, \tau \mid \bar{\sigma}, x, \bar{\tau}\} & -\{\sigma, m+1, n-1, \tau \mid \bar{\sigma}, x, \bar{\tau}\} \\
=\quad\{\sigma, m, n, \tau \mid \bar{\sigma}, n, \bar{\tau}\} & \\
& +\left\{\sigma, m+1, n-1+\tau_{1}, \tau \backslash \tau_{1} \mid \bar{\sigma}, n, \bar{\tau} \backslash \bar{\tau}_{1}\right\}
\end{aligned}
$$

If $t=0$ then omit the second term.

Proof This is the special case

$$
K_{\tau, \bar{\tau}}^{\sigma, \bar{\sigma}}(m, n \mid x)-K_{\tau, \bar{\tau}}^{\sigma, \bar{\sigma}}(m+1, n-1 \mid x)=K_{\tau, \bar{\tau}}^{\sigma, \bar{\sigma}}(m, n \mid n)-K_{\tau, \bar{\tau}}^{\sigma, \bar{\sigma}}(m+1, n-1 \mid n)
$$

of Lemma 2.3, rewritten using (2.2). Note that the terms on the left are each the first case of (2.2), and the terms on the right are the first and third cases, respectively.

Corollary 2.9 Assume $\sigma, \tau, \bar{\sigma}, \bar{\tau}$ satisfy Hypothesis 2.1 . If $0<m \leq n$ then

$$
\begin{aligned}
& \{\sigma, m, n, \tau \mid \bar{\sigma}, m-1, \bar{\tau}\}-\{\sigma, n+1, m-1, \tau \mid \bar{\sigma}, m-1, \bar{\tau}\} \\
& =\{\sigma, m, n, \tau \mid \bar{\sigma}, m, \bar{\tau}\} \\
& +\left\{\sigma, n+1, m-1+\tau_{1}, \tau \backslash \tau_{1} \mid \bar{\sigma}, m, \bar{\tau} \backslash \bar{\tau}_{1}\right\} .
\end{aligned}
$$

If $t=0$ then omit the second term. 
Proof This is the special case

$$
\begin{aligned}
& K_{\tau, \bar{\tau}}^{\sigma, \bar{\sigma}}(m, n \mid m-1)-K_{\tau, \bar{\tau}}^{\sigma, \bar{\sigma}}(n+1, m-1 \mid m-1) \\
& \quad=K_{\tau, \bar{\tau}}^{\sigma, \bar{\sigma}}(m, n \mid m)-K_{\tau, \bar{\tau}}^{\sigma, \bar{\sigma}}(n+1, m-1 \mid m)
\end{aligned}
$$

of Lemma 2.3, rewritten using (2.2). Note that the terms on the left are each the first case of (2.2), and the terms on the right are the first and third cases, respectively.

Corollary 2.10 Assume $\sigma, \tau, \bar{\sigma}, \bar{\tau}$ satisfy Hypothesis 2.1 . If $0 \leq m<n$ then

$$
\begin{aligned}
\{\sigma, m, n, \tau \mid \bar{\sigma}, m, \bar{\tau}\} & -\{\sigma, n, m, \tau \mid \bar{\sigma}, m, \bar{\tau}\} \\
=\quad- & \left\{\sigma \backslash \sigma_{s}, m+\sigma_{s}, n, \tau \mid \bar{\sigma} \backslash \bar{\sigma}_{s}, m+1, \bar{\tau}\right\} \\
+ & \left\{\sigma, n, m+\tau_{1}, \tau \backslash \tau_{1} \mid \bar{\sigma}, m+1, \bar{\tau} \backslash \bar{\tau}_{1}\right\}
\end{aligned}
$$

If $s=0$ then omit the first term. Similarly, if $t=0$ then omit the second term.

Proof This is the special case

$$
K_{\tau, \bar{\tau}}^{\sigma, \bar{\sigma}}(m, n \mid m)-K_{\tau, \bar{\tau}}^{\sigma, \bar{\sigma}}(n, m \mid m)=K_{\tau, \bar{\tau}}^{\sigma, \bar{\sigma}}(m, n \mid m+1)-K_{\tau, \bar{\tau}}^{\sigma, \bar{\sigma}}(n, m \mid m+1)
$$

of Lemma 2.3, rewritten using (2.2). Note that the terms on the left are each the first case of (2.2), and the terms on the right are the second and third cases, respectively.

\subsection{Fundamental Schur positive difference expression}

We are now close to proving our main theorem, Theorem 2.13. The following two lemmas, which are consequences of the above four corollaries, facilitate the proof of the equation therein, (2.24).

Lemma 2.11 Assume $\sigma, \tau, \bar{\sigma}, \bar{\tau}$ satisfy Hypothesis 2.1. If $M \geq 1, a \geq 2$ and $k \geq 1$ then

$$
\begin{aligned}
& \left\{\sigma, k a, k a, a^{M}, \tau \mid \bar{\sigma},(k-1) a+1,1^{M}, \bar{\tau}\right\} \\
& -\left\{\sigma, k a+1, k a-1, a^{M}, \tau \mid \bar{\sigma},(k-1) a+1,1^{M}, \bar{\tau}\right\} \\
& +\sum_{i=1}^{M}\left(\left\{\sigma, a+1, a^{i-1}, k a-1, k a, a^{M-i}, \tau \mid \bar{\sigma}, 1^{i},(k-1) a+1,1^{M-i}, \bar{\tau}\right\}\right. \\
& \left.\quad-\left\{\sigma, a+1, a^{i-1}, k a, k a-1, a^{M-i}, \tau \mid \bar{\sigma}, 1^{i},(k-1) a+1,1^{M-i}, \bar{\tau}\right\}\right) \\
& =\left\{\sigma, k a, k a, a^{M}, \tau \mid \bar{\sigma}, k a, 1^{M}, \bar{\tau}\right\} \\
& \quad+\left\{\sigma, k a+1,(k+1) a-1, a^{M-1}, \tau \mid \bar{\sigma}, k a, 1^{M-1}, \bar{\tau}\right\} \\
& \quad-\left\{\sigma,(k+1) a, k a, a^{M-1}, \tau \mid \bar{\sigma}, k a, 1^{M-1}, \bar{\tau}\right\}
\end{aligned}
$$




$$
\begin{aligned}
& +\left\{\sigma, a+1, a^{M-1}, k a, k a-1+\tau_{1}, \tau \backslash \tau_{1} \mid \bar{\sigma}, 1^{M}, k a, \bar{\tau} \backslash \bar{\tau}_{1}\right\} \\
& +\sum_{i=1}^{M-1}\left(\left\{\sigma, a+1, a^{i-1}, k a,(k+1) a-1, a^{M-1-i}, \tau \mid \bar{\sigma}, 1^{i}, k a, 1^{M-i-1}, \bar{\tau}\right\}\right. \\
& \left.\quad-\left\{\sigma, a+1, a^{i-1},(k+1) a-1, k a, a^{M-1-i}, \tau \mid \bar{\sigma}, 1^{i}, k a, 1^{M-i-1}, \bar{\tau}\right\}\right)
\end{aligned}
$$

where the fourth term on the right is to be omitted if $t=0$.

Proof The first two terms on the right result from applying Corollary 2.8 to the first two terms on the left, using $m=n=k a$ and $x=(k-1) a+1$. The other terms result from applying Corollary 2.7 to each summand on the left side, in turn, using $m=k a-1$ and $x=(k-1) a+1$. The positive term in the $i$ th summand on the right is the positive term of (2.20) applied to the $i$ th summand on the left, and the negative term in the $i$ th summand on the right is the negative term of (2.20) applied to the $(i+1)$ th summand on the left. The third term on the right is the negative term of (2.20) applied to the first summand on the left, and the fourth term on the right is the positive term of (2.20) applied to the $M$ th summand on the left.

Lemma 2.12 Assume $\sigma, \tau, \bar{\sigma}, \bar{\tau}$ satisfy Hypothesis 2.1. If $M \geq 1, a \geq 2$ and $k \geq 1$ then

$$
\begin{aligned}
& \left\{\sigma, k a+1,(k+1) a-1, a^{M}, \tau \mid \bar{\sigma}, k a, 1^{M}, \bar{\tau}\right\} \\
& -\left\{\sigma,(k+1) a, k a, a^{M}, \tau \mid \bar{\sigma}, k a, 1^{M}, \bar{\tau}\right\} \\
& +\sum_{i=1}^{M}\left(\left\{\sigma, a+1, a^{i-1}, k a,(k+1) a-1, a^{M-i}, \tau \mid \bar{\sigma}, 1^{i}, k a, 1^{M-i}, \bar{\tau}\right\}\right. \\
& \left.\quad-\left\{\sigma, a+1, a^{i-1},(k+1) a-1, k a, a^{M-i}, \tau \mid \bar{\sigma}, 1^{i}, k a, 1^{M-i}, \bar{\tau}\right\}\right) \\
& =\left\{\sigma, k a+1,(k+1) a-1, a^{M}, \tau \mid \bar{\sigma}, k a+1,1^{M}, \bar{\tau}\right\} \\
& +\left\{\sigma,(k+1) a,(k+1) a, a^{M-1}, \tau \mid \bar{\sigma}, k a+1,1^{M-1}, \bar{\tau}\right\} \\
& \quad-\left\{\sigma,(k+1) a+1,(k+1) a-1, a^{M-1}, \tau \mid \bar{\sigma}, k a+1,1^{M-1}, \bar{\tau}\right\} \\
& \quad+\left\{\sigma, a+1, a^{M-1},(k+1) a-1, k a+\tau_{1}, \tau \backslash \tau_{1} \mid \bar{\sigma}, 1^{M}, k a+1, \bar{\tau} \backslash \bar{\tau}_{1}\right\} \\
& \quad+\sum_{i=1}^{M-1}\left(\left\{\sigma, a+1, a^{i-1},(k+1) a-1,(k+1) a, a^{M-1-i}, \tau\right.\right. \\
& \left.\quad \mid \bar{\sigma}, 1^{i}, k a+1,1^{M-i-1}, \bar{\tau}\right\} \\
& \quad-\left\{\sigma, a+1, a^{i-1},(k+1) a,(k+1) a-1, a^{M-1-i}, \tau\right. \\
& \left.\left.\quad \mid \bar{\sigma}, 1^{i}, k a+1,1^{M-i-1}, \bar{\tau}\right\}\right) \\
& \quad
\end{aligned}
$$


where the fourth term on the right is to be omitted if $t=0$.

Proof The first two terms on the right result from applying Corollary 2.9 to the first two terms on the left, using $m=k a+1$ and $n=(k+1) a-1$. The other terms result from applying Corollary 2.10 to each summand on the left side, in turn, using $m=k a$ and $n=(k+1) a-1$. The positive term in the $i$ th summand on the right is the positive term of (2.23) applied to the $i$ th summand on the left, and the negative term in the $i$ th summand on the right is the negative term of (2.23) applied to the $(i+1)$ th summand on the left. The third term on the right is the negative term of (2.23) applied to the first summand on the left, and the fourth term on the right is the positive term of (2.23) applied to the $M$ th summand on the left.

We now come to our main theorem.

Theorem 2.13 Assume $\sigma, \tau, \bar{\sigma}, \bar{\tau}$ satisfy Hypothesis 2.1. Then, for $a \geq 2$ and $n \geq 2$

$$
\begin{aligned}
& \left\{\sigma, a^{n}, \tau \mid \bar{\sigma}, 1^{n-1}, \bar{\tau}\right\}-\left\{\sigma, a+1, a^{n-2}, a-1, \tau \mid \bar{\sigma}, 1^{n-1}, \bar{\tau}\right\} \\
& =\sum_{k=1}^{\left\lfloor\frac{n}{2}\right\rfloor}\left\{\sigma, k a, k a, a^{n-2 k}, \tau \mid \bar{\sigma}, k a, 1^{n-2 k}, \bar{\tau}\right\} \\
& + \\
& +\sum_{k=1}^{\left.\frac{n-1}{2}\right\rfloor}\left\{\sigma, a+1, a^{n-2 k-1}, k a, k a-1+\tau_{1}, \tau \backslash \tau_{1} \mid \bar{\sigma}, 1^{n-2 k}, k a, \bar{\tau} \backslash \bar{\tau}_{1}\right\} \\
& +\sum_{k=1}^{\left.\frac{n-1}{2}\right\rfloor}\left\{\sigma, k a+1,(k+1) a-1, a^{n-2 k-1}, \tau \mid \bar{\sigma}, k a+1,1^{n-2 k-1}, \bar{\tau}\right\} \\
& +\sum_{k=1}^{\left.\frac{n-2}{2}\right\rfloor}\left\{\sigma, a+1, a^{n-2 k-2},(k+1) a-1, k a+\tau_{1}, \tau \backslash \tau_{1}\right.
\end{aligned}
$$

If $s=0$, then omit the $\sigma$ from each expression. If $t=0$, then omit all terms containing $\tau \backslash \tau_{1}$ or $\bar{\tau} \backslash \bar{\tau}_{1}$. If $t=1$, then just omit $\tau \backslash \tau_{1}$ and $\bar{\tau} \backslash \bar{\tau}_{1}$ wherever they occur. The right side of (2.24) then has $2 n-2$ terms when $t>0$, and $n-1$ terms when $t=0$.

Example 2.14 Before we prove this relation we provide four examples involving ribbon Schur functions. Note how the results differ for the cases $s=0=t, s \neq 0=t$, 
$s=0 \neq t$ and $s \neq 0 \neq t$.

$$
\begin{aligned}
& r_{(2,2,2)}-r_{(3,2,1)}=\{2,2,2 \mid 2,1\}+\{3,3 \mid 3\} . \\
& r_{(3,2,2,2)}-r_{(3,3,2,1)}=\{3,2,2,2 \mid 1,2,1\}+\{3,3,3 \mid 1,3\} . \\
& r_{(2,2,2,1)}-r_{(3,2,1,1)}=\{2,2,2,1 \mid 2,1,1\}+\{3,2,2 \mid 1,2\} \\
& +\{3,3,1 \mid 3,1\}+\{4,3 \mid 3\} \text {. } \\
& r_{(3,2,2,2,1)}-r_{(3,3,2,1,1)}=\{3,2,2,2,1 \mid 1,2,1,1\}+\{3,3,2,2 \mid 1,1,2\} \\
& +\{3,3,3,1 \mid 1,3,1\}+\{3,4,3 \mid 1,3\} \text {. }
\end{aligned}
$$

Proof of Theorem 2.13 To obtain expression (2.24) we first write

$$
\begin{aligned}
& \left\{\sigma, a^{n}, \tau \mid \bar{\sigma}, 1^{n-1}, \bar{\tau}\right\}-\left\{\sigma, a+1, a^{n-2}, a-1, \tau \mid \bar{\sigma}, 1^{n-1}, \bar{\tau}\right\} \\
& =\left\{\sigma, a^{n}, \tau \mid \bar{\sigma}, 1^{n-1}, \bar{\tau}\right\}-\left\{\sigma, a+1, a-1, a^{n-2}, \tau \mid \bar{\sigma}, 1^{n-1}, \bar{\tau}\right\} \\
& +\sum_{i=1}^{n-2}\left(\left\{\sigma, a+1, a^{i-1}, a-1, a, a^{n-2-i}, \tau \mid \bar{\sigma}, 1^{n-1}, \bar{\tau}\right\}\right. \\
& \left.-\left\{\sigma, a+1, a^{i-1}, a, a-1, a^{n-2-i}, \tau \mid \bar{\sigma}, 1^{n-1}, \bar{\tau}\right\}\right) .
\end{aligned}
$$

Now, starting with Lemma 2.11 in the case $M=n-2$ and $k=1$, we repeatedly apply Lemmas 2.11 and 2.12 alternately. Each application of Lemma 2.11 decreases $M$ by 1 but leaves $k$ fixed, and each application of Lemma 2.12 decreases $M$ by 1 and increases $k$ by 1 . The final application is made with $M=1$, and is of Lemma 2.11 with $k=\frac{n-1}{2}$ if $n$ is odd, and of Lemma 2.12 with $k=\frac{n-2}{2}$ if $n$ is even.

Following each application, the first and fourth terms on the right side of the expression in Lemma 2.11 or Lemma 2.12 contribute to the final expression (2.24). The remaining terms (second, third and the summation) are then acted upon by the subsequent application of Lemma 2.11 or Lemma 2.12. When applying Lemma 2.11, the first and fourth terms on the right give rise to the $k$ th summands in the first and second terms on the right of (2.24). The summands $1 \leq k \leq\left\lfloor\frac{n-1}{2}\right\rfloor$ of these latter two terms arise in this way. Similarly, when applying Lemma 2.12, the first and fourth terms on the right give rise to the $k$ th summands in the third and fourth terms on the right of (2.24). The summands $1 \leq k \leq\left\lfloor\frac{n-2}{2}\right\rfloor$ of these latter two terms arise in this way.

If $n$ is odd, the final application of Lemma 2.11 is made with $M=1$ and $k=$ $\frac{n-1}{2}$. It remains to deal with the second and third terms that arise on the right of the expression in Lemma 2.11. This is accomplished using Corollary 2.9, which yields

$$
\begin{gathered}
\{\sigma, k a+1,(k+1) a-1, \tau \mid \bar{\sigma}, k a, \bar{\tau}\}-\{\sigma,(k+1) a, k a, \tau \mid \bar{\sigma}, k a, \bar{\tau}\} \\
=\{\sigma, k a+1,(k+1) a-1, \tau \mid \bar{\sigma}, k a+1, \bar{\tau}\} \\
\quad+\left\{\sigma,(k+1) a, k a+\tau_{1}, \tau \backslash \tau_{1} \mid \bar{\sigma}, k a+1, \bar{\tau} \backslash \bar{\tau}_{1}\right\}
\end{gathered}
$$

The first term on the right here gives the additional $k=\frac{n-1}{2}$ summand of the third term on the right of (2.24), and the second term gives the odd $n$ case of the final term. 
If $n$ is even, the final application of Lemma 2.12 is made with $M=1$ and $k=$ $\frac{n-2}{2}$. It remains to deal with the second and third terms that arise on the right of the expression in Lemma 2.12. This is accomplished using Corollary 2.8, which yields

$$
\begin{aligned}
& \{\sigma,(k+1) a,(k+1) a, \tau \mid \bar{\sigma}, k a+1, \bar{\tau}\} \\
& -\{\sigma,(k+1) a+1,(k+1) a-1, \tau \mid \bar{\sigma}, k a+1, \bar{\tau}\} \\
& \quad=\{\sigma,(k+1) a,(k+1) a, \tau \mid \bar{\sigma},(k+1) a, \bar{\tau}\} \\
& \quad+\left\{\sigma,(k+1) a+1,(k+1) a-1+\tau_{1}, \tau \backslash \tau_{1} \mid \bar{\sigma},(k+1) a, \bar{\tau} \backslash \bar{\tau}_{1}\right\} .
\end{aligned}
$$

The first term on the right here gives the additional $k=\frac{n}{2}$ summand of the first term on the right of (2.24), and the second term gives the even $n$ case of the final term. This completes the proof of (2.24).

\section{Schur positivity of ribbon and skew Schur functions}

We now apply our results from the previous section to derive some new differences of skew Schur functions that are Schur positive.

The crucial feature of the formulae (2.21) and (2.24) for the differences of certain skew Schur functions is that they are expressed as wholly positive sums of skew Schur functions, each of which is itself necessarily Schur positive. This leads immediately to the following theorem.

Theorem 3.1 Assume $\sigma, \tau, \bar{\sigma}, \bar{\tau}$ satisfy Hypothesis 2.1. If $a \geq b \geq 2$ then

$$
\{\sigma, a, b, \tau \mid \bar{\sigma}, 1, \bar{\tau}\}-\{\sigma, a+1, b-1, \tau \mid \bar{\sigma}, 1, \bar{\tau}\}
$$

and

$$
\left\{\sigma, a^{n}, \tau \mid \bar{\sigma}, 1^{n-1}, \bar{\tau}\right\}-\left\{\sigma, a+1, a^{n-2}, a-1, \tau \mid \bar{\sigma}, 1^{n-1}, \bar{\tau}\right\}
$$

are Schur positive, where $\{\alpha \mid \beta\}$ denotes the skew Schur function $s_{\lambda / \mu}$ satisfying $r^{(1)}(\lambda / \mu)=\alpha$ and $r^{(2)}(\lambda / \mu)=\beta$.

The descent set of a SYT, $T$, is the set of all entries $i$ such that $i+1$ appears in a lower row than $i$. With this in mind we recall the following relationship between ribbon Schur functions $r_{\alpha}$ for $\alpha \vDash N$, and Schur functions $s_{\lambda}$ for $\lambda \vdash N$.

Lemma 3.2 [4, Theorem 7] Let $\alpha \vDash N$ then

$$
r_{\alpha}=\sum_{\lambda \vdash N} d_{\lambda \alpha} s_{\lambda}
$$

where $d_{\lambda \alpha}$ is the number of SYTX of shape $\lambda$ and descent set $S(\alpha)$.

This lemma, together with Theorem 3.1, allows us to prove the following result first conjectured by McNamara [10], which is analogous to Theorem 1.4. 
Theorem 3.3 Let $\lambda, \mu \vdash N$ then

$$
r_{\mu}-r_{\lambda}
$$

is Schur positive if and only if $\mu \leq_{\text {dom }} \lambda$ and $\ell(\lambda)=\ell(\mu)$.

Proof First we prove that if $\mu \bigsqcup_{d o m} \lambda$ or $\ell(\lambda) \neq \ell(\mu)$ then $r_{\mu}-r_{\lambda}$ is not Schur positive. Note that by Lemma 3.2 if $S(\lambda)=\left\{i_{1}, i_{2}, \ldots, i_{\ell(\lambda)-1}\right\}$ then in any SYT, $T$, of shape $v$ and descent set $S(\lambda)$ contributing towards the coefficient of $s_{v}$ in the Schur function expansion of $r_{\lambda}$, the entries $1, \ldots, i_{j}$ must appear in the top $j$ rows of $T$. Moreover, $s_{\lambda}$ appears in the Schur function expansion of $r_{\lambda}$ with positive coefficient due to the SYT

$$
\begin{array}{ccc}
1 & 2 & 3 \\
i_{1}+1 & \cdots & i_{2} \\
\vdots & \ddots & \\
i_{\ell(\lambda)-1}+1 & \cdots & N
\end{array}
$$

Consequently, if $\mu \leq$ dom $_{\text {d }} \lambda$ then for some $i$ we have

$$
\lambda_{1}+\cdots+\lambda_{i}<\mu_{1}+\cdots+\mu_{i}
$$

and there cannot exist a SYT of shape $\lambda$ and descent set $S(\mu)$ because the numbers $1, \ldots, \mu_{1}+\cdots+\mu_{i}$ must appear in only $\lambda_{1}+\cdots+\lambda_{i}$ boxes. Hence by Lemma 3.2 it follows that $s_{\lambda}$ is a term in the Schur function expansion of $r_{\lambda}$ but not $r_{\mu}$ and so $r_{\mu}-r_{\lambda}$ is not Schur positive.

Now note that if $\mu \leq{ }_{d o m} \lambda$ then since

$$
\lambda_{1}+\cdots+\lambda_{i} \geq \mu_{1}+\cdots+\mu_{i}
$$

for all $i$ it follows that $\ell(\lambda) \leq \ell(\mu)$. We need to show that if $\ell(\lambda)<\ell(\mu)$ then $r_{\mu}-r_{\lambda}$ is not Schur positive. Observe by Lemma 3.2 that if $\lambda=\left(\rho, 1^{a}\right)$ where $\rho$ is a partition with $\rho_{\ell(\rho)}>1$ and $a>0$ then $S(\lambda)=\left\{i_{1}, i_{2}, \ldots, i_{\ell(\rho)}, N-a+1, \ldots, N-1\right\}$ and the lexicographically greatest partition appearing as an index of a Schur function in the Schur function expansion of $r_{\lambda}$ is $\phi(\lambda)=\left(N-\ell(\rho)-a+1, \ell(\rho), 1^{a-1}\right)=$ $\left(N-\ell(\lambda)+1, \ell(\rho), 1^{a-1}\right)$ due to the SYT

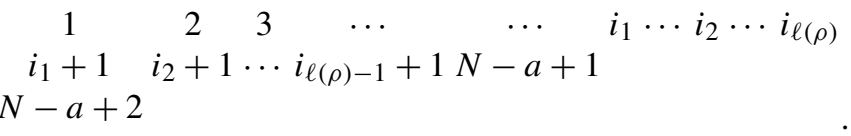

$$
\begin{aligned}
& \text { N }
\end{aligned}
$$

If $a=0$ then $S(\lambda)=\left\{i_{1}, i_{2}, \ldots, i_{\ell(\rho)-1}\right\}$ and Lemma 3.2 shows the lexicographically greatest partition appearing as an index of a Schur function in the Schur function expansion of $r_{\lambda}$ is $\phi(\lambda)=(N-\ell(\rho)+1, \ell(\rho)-1)=(N-\ell(\lambda)+1, \ell(\rho)-1)$ due to the SYT

$$
\begin{array}{cccc}
1 & 2 & 3 & \cdots \\
i_{1}+1 & i_{2}+1 & \cdots & i_{\ell(\rho)-1}+1
\end{array} i_{1} \cdots i_{2} \cdots N
$$


Consequently, if $\ell(\lambda)<\ell(\mu)$ we have $\phi(\mu)<_{\text {lex }} \phi(\lambda)$ and so $s_{\phi(\lambda)}$ is a term in the Schur function expansion of $r_{\lambda}$ but not $r_{\mu}$ and so $r_{\mu}-r_{\lambda}$ is not Schur positive.

Now we prove that if $\mu \leq_{d o m} \lambda$ and $\ell(\lambda)=\ell(\mu)$ then $r_{\mu}-r_{\lambda}$ is Schur positive. Note we only need to show that the result holds for each of the types of cover relation in the dominance order with $\ell(\lambda)=\ell(\mu)=\ell$. For the first cover relation, if $\lambda=$ $(\sigma, a+1, b-1, \tau), \mu=(\sigma, a, b, \tau)$ and $\sigma=\left(\sigma_{1}, \ldots, \sigma_{s}\right), \tau=\left(\tau_{1}, \ldots, \tau_{t}\right)$ with $\sigma_{s} \geq$ $a+1, b-1 \geq \tau_{1}$ and $a \geq b \geq 2$ then $r_{\mu}-r_{\lambda}$ is Schur positive by Theorem 3.1 with $r_{\mu}=\left\{\sigma, a, b, \tau \mid 1^{\ell-1}\right\}$ and $r_{\lambda}=\left\{\sigma, a+1, b-1, \tau \mid 1^{\ell-1}\right\}$. For the second cover relation, if $\lambda=\left(\sigma, a+1, a^{n-2}, a-1, \tau\right), \mu=\left(\sigma, a^{n}, \tau\right)$ and $\sigma=\left(\sigma_{1}, \ldots, \sigma_{s}\right), \tau=$ $\left(\tau_{1}, \ldots, \tau_{t}\right)$ with $\sigma_{s} \geq a+1, a-1 \geq \tau_{1}$ and $n \geq 2$ then $r_{\mu}-r_{\lambda}$ is Schur positive by Theorem 3.1 with $r_{\mu}=\left\{\sigma, a^{n}, \tau \mid 1^{\ell-1}\right\}$ and $r_{\lambda}=\left\{\sigma, a+1, a^{n-2}, a-1, \tau \mid 1^{\ell-1}\right\}$. The proof is now complete.

\section{Application to Schubert calculus}

In view of the well established link between the algebra of Schur functions and Schubert calculus $[11,15]$, it is appropriate to examine the consequences of Theorem 3.1 in the Schubert calculus context.

Let $\operatorname{Gr}\left(\ell, \mathbb{C}^{m}\right)$ denote the Grassmannian of $\ell$-dimensional subspaces in $\mathbb{C}^{m}$. The cohomology ring $H^{*}\left(G r\left(\ell, \mathbb{C}^{m}\right), \mathbb{Z}\right)$ has an additive basis of Schubert classes, $\sigma_{\lambda}$, indexed by partitions $\lambda \subseteq\left(k^{\ell}\right)$, where $k=m-\ell$. The product rule for Schubert classes in $H^{*}\left(G r\left(\ell, \mathbb{C}^{m}\right), \mathbb{Z}\right)$ takes the following form, see for example [11]

$$
\sigma_{\lambda} \cdot \sigma_{\mu}=\sum_{\nu \subseteq\left(k^{\ell}\right)} c_{\lambda \mu}^{\nu} \sigma_{\nu}
$$

This differs from the product rule for Schur functions

$$
s_{\lambda} \cdot s_{\mu}=\sum_{v} c_{\lambda \mu}^{v} s_{v}
$$

only in the restriction placed on the partitions $v$. The coefficients in both products are identical, namely the familiar non-negative integer Littlewood-Richardson coefficients.

One way to evaluate the product of two Schubert classes is to proceed by Schur function methods in a manner that automatically restricts partitions $v$ to those satisfying the constraint $v \subseteq\left(k^{\ell}\right)$. To this end, consider the skew Schur function $s_{\left(k^{\ell}\right) / v}$. For any partition $v \subseteq\left(k^{\ell}\right)$, we let $v^{c}$ denote the partition complementary to $v$ in an $\ell \times k$ rectangle, which is to say

$$
v^{c}{ }_{i}=k-v_{\ell-i+1} \quad \text { for } i=1,2 \ldots, \ell .
$$

Diagrammatically, the Young diagram of shape $v^{c}$ is obtained by rotating that of shape $\left(k^{\ell}\right) / v$ through $180^{\circ}$. For example, if $\ell=4, k=5$ and $v=(5,4,2,1)$, then 
$\nu^{c}=(4,3,1)$ as illustrated by

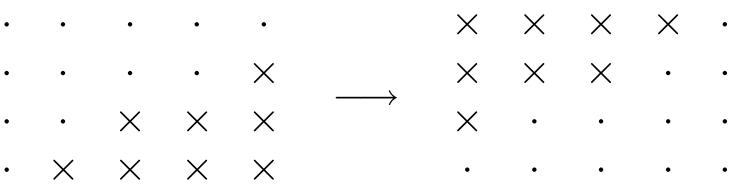

where $\times$ indicates a box, and $\cdot$ no box.

With this definition of $v^{c}$, we have

$$
s_{\left(k^{\ell}\right) / v}= \begin{cases}s_{v^{c}} & \text { if } v \subseteq\left(k^{\ell}\right) ; \\ 0 & \text { otherwise. }\end{cases}
$$

The first case in (4.3) is [15, Exercise 7.56(a)]. The other case in (4.3) is perhaps obvious, but may be seen formally by noting that the definition of skew Schur functions [9] implies that

$$
\left\langle s_{\left(k^{\ell}\right) / v}, s_{\rho}\right\rangle=\left\langle s_{\left(k^{\ell}\right)}, s_{v} s_{\rho}\right\rangle
$$

for all $\rho$, where the bilinear form $\langle\cdot, \cdot\rangle$ on symmetric functions is such that $\left\langle s_{\lambda}, s_{\mu}\right\rangle=$ $\delta_{\lambda \mu}$. However, it follows from the Littlewood-Richardson rule that the right-hand side is zero for all $\rho$ if $\nu \nsubseteq\left(k^{\ell}\right)$. Hence, in such a case $s_{\left(k^{\ell}\right) / v}=0$, as claimed in (4.3).

Now consider the skew Schur function $s_{\mu^{c}} / \lambda$. This can be expanded in terms of Schur functions in two ways as follows:

$$
s_{\mu^{c} / \lambda}=\sum_{\rho} c_{\lambda \rho}^{\mu^{c}} s_{\rho}
$$

and

$$
\begin{aligned}
& s_{\mu^{c} / \lambda}=\sum_{\kappa}\left\langle s_{\mu^{c} / \lambda}, s_{\kappa}\right\rangle s_{\kappa} \quad=\sum_{\kappa}\left\langle s_{\mu^{c}}, s_{\kappa} s_{\lambda}\right\rangle s_{\kappa} \\
& =\sum_{\kappa}\left\langle s_{\left(k^{\ell}\right) / \mu}, s_{\kappa} s_{\lambda}\right\rangle s_{\kappa} \quad=\sum_{\kappa}\left\langle s_{\left(k^{\ell}\right)}, s_{\kappa} s_{\lambda} s_{\mu}\right\rangle s_{\kappa} \\
& =\sum_{\kappa} \sum_{v} c_{\lambda \mu}^{v}\left\langle s_{\left(k^{\ell}\right)}, s_{\kappa} s_{\nu}\right\rangle s_{\kappa}=\sum_{\kappa} \sum_{v} c_{\lambda \mu}^{v}\left\langle s_{\left(k^{\ell}\right) / v}, s_{\kappa}\right\rangle s_{\kappa} \\
& =\sum_{\kappa} \sum_{\nu \subseteq\left(k^{\ell}\right)} c_{\lambda \mu}^{\nu}\left\langle s_{\nu^{c}}, s_{\kappa}\right\rangle s_{\kappa}=\sum_{\nu \subseteq\left(k^{\ell}\right)} c_{\lambda \mu}^{\nu} s_{\nu^{c}} .
\end{aligned}
$$

On comparing these two expressions, it can be seen that to evaluate the product $\sigma_{\lambda} \cdot \sigma_{\mu}$ of Schubert classes given in (4.1) one merely has to expand the skew Schur function $s_{\mu^{c} / \lambda}$ in terms of Schur functions. To be more precise

$$
\sigma_{\lambda} \cdot \sigma_{\mu}=\sum_{\nu} c_{\lambda, v^{c}}^{\mu^{c}} \sigma_{v}
$$

as proved also in [6] and implicitly in [16].

Moreover, the skew shape corresponding to $\mu^{c} / \lambda$ is that of the diagram obtained by placing the Young diagram of shape $\lambda$ in the top lefthand corner of an $\ell \times k$ 
rectangle, and that of $\mu$ rotated through $180^{\circ}$ in the bottom righthand corner and deleting both sets of boxes from the Young diagram of $\left(k^{\ell}\right)$. This is illustrated in the case $\ell=5, k=6, \lambda=(5,2,1)$ and $\mu=(5,4,2)$ as shown below, where each $\lambda$ signifies a box of the Young diagram of shape $\lambda$, each $\mu$ a box of the rotated Young diagram of shape $\mu$.

$$
\begin{aligned}
& \lambda \lambda \lambda \lambda \lambda \alpha \\
& \lambda \lambda \alpha \alpha \alpha \alpha \\
& \lambda \alpha \alpha \alpha \mu \mu \\
& \alpha \alpha \mu \mu \mu \mu \\
& \alpha \mu \mu \mu \mu \mu
\end{aligned}
$$

In a case, such as this, where the Young diagrams of shape $\lambda$ and $\mu$ do not overlap, so that $s_{\mu^{c} / \lambda}$ is non-zero, the remaining boxes labelled $\alpha$ are those of the required skew shape $\mu^{c} / \lambda$. In this example, we have $s_{\mu^{c} / \lambda}=s_{(6,6,4,2,1) /(5,2,1)}$, which in the overlap notation of $(1.5)$ is $\{1,4,3,2,1 \mid 1,2,1,1\}$.

As a direct consequence of this, we are in a position to apply our previous results to the question of what we shall call the Schubert positivity of differences of products of Schubert classes. In this connection we make the following definition.

Definition 4.1 If an element $\sigma \in H^{*}\left(G r\left(\ell, \mathbb{C}^{m}\right), \mathbb{Z}\right)$ can be written as a non-negative linear combination of Schubert classes then we say that $\sigma$ is Schubert positive.

Then we have the following theorem on differences of products of Schubert classes.

Theorem 4.2 Let $\sigma, \tau, \bar{\sigma}, \bar{\tau}$ satisfy Hypothesis 2.1 , and let the partitions $\lambda, \mu, \kappa, \rho \subseteq$ $\left(k^{\ell}\right)$ be such that in the overlap notation of (1.1) either

$$
\begin{aligned}
& \mu^{c} / \lambda=(\sigma, a, b, \tau \mid \bar{\sigma}, 1, \bar{\tau}) \\
& \rho^{c} / \kappa=(\sigma, a+1, b-1, \tau \mid \bar{\sigma}, 1, \bar{\tau})
\end{aligned}
$$

with $a \geq b \geq 2$, or

$$
\begin{aligned}
& \mu^{c} / \lambda=\left(\sigma, a^{n}, \tau \mid \bar{\sigma}, 1^{n-1}, \bar{\tau}\right) \\
& \rho^{c} / \kappa=\left(\sigma, a+1, a^{n-2}, a-1, \tau \mid \bar{\sigma}, 1^{n-1}, \bar{\tau}\right)
\end{aligned}
$$

with $a \geq 2$, then the product of Schubert classes in $H^{*}\left(G r\left(\ell, \mathbb{C}^{k+l}\right), \mathbb{Z}\right)$ is such that

$$
\sigma_{\lambda} \cdot \sigma_{\mu}-\sigma_{\kappa} \cdot \sigma_{\rho}
$$

is Schubert positive.

Proof Theorem 3.1 implies that in each case $s_{\mu^{c} / \lambda}-s_{\rho^{c} / \kappa}$ is Schur positive, which is to say $c_{\lambda, v^{c}}^{\mu^{c}}-c_{\kappa, v^{c}}^{\rho^{c}}$ is non-negative for each $v$. The required result then follows from (4.4).

As a further application, it is instructive to consider the implications of our Theorem 3.3 on differences of ribbon Schur functions. 
Theorem 4.3 Let the partitions $\lambda, \mu, \kappa, \rho \subseteq\left(k^{\ell}\right)$ be such that $\mu^{c} / \lambda=\left(\alpha \mid 1^{\ell-1}\right)$ and $\rho^{c} / \kappa=\left(\beta \mid 1^{\ell-1}\right)$ with $\alpha$ and $\beta$ partitions of lengths $\ell(\alpha)=\ell(\beta)=\ell$ and $|\alpha|=$ $|\beta|=N \leq \ell+k-1$, such that $\alpha \leq{ }_{\text {dom }} \beta$. Then the difference of products of Schubert classes

$$
\sigma_{\lambda} \cdot \sigma_{\mu}-\sigma_{\kappa} \cdot \sigma_{\rho}
$$

is Schubert positive in $H^{*}\left(G r\left(\ell, \mathbb{C}^{k+l}\right), \mathbb{Z}\right)$.

Proof Since $\mu^{c} / \lambda=\left(\alpha \mid 1^{\ell-1}\right)$ is a ribbon, it follows from Lemma 3.2 that

$$
r_{\alpha}=s_{\mu^{c} / \lambda}=\sum_{\nu \subseteq\left(k^{\ell}\right)} c_{\lambda, \nu^{c}}^{\mu^{c}} s_{\nu^{c}}=\sum_{\nu \subseteq\left(k^{\ell}\right)} d_{\nu^{c} \alpha} s_{\nu^{c}} .
$$

Using this in (4.4) gives

$$
\sigma_{\lambda} \cdot \sigma_{\mu}=\sum_{\nu \subseteq\left(k^{l}\right)} d_{\nu^{c} \alpha} \sigma_{\nu}
$$

A similar result applies to $\sigma_{\kappa} \cdot \sigma_{\rho}$, so that from (4.4) we obtain

$$
\sigma_{\lambda} \cdot \sigma_{\mu}-\sigma_{\kappa} \cdot \sigma_{\rho}=\sum_{\nu \subseteq\left(k^{\ell}\right)}\left(d_{\nu^{c} \alpha}-d_{\nu^{c} \beta}\right) \sigma_{\nu} .
$$

By hypothesis, $|\alpha|=|\beta|=N, \ell(\alpha)=\ell(\beta)=\ell$ and $\alpha \leq_{\text {dom }} \beta$, so that Theorem 3.3 implies that

$$
r_{\alpha}-r_{\beta}=\sum_{\eta}\left(d_{\eta \alpha}-d_{\eta \beta}\right) s_{\eta}
$$

is Schur positive, and thus $d_{\eta \alpha}-d_{\eta \beta} \geq 0$ for all $\eta$. This ensures, in turn, that $\sigma_{\lambda} \cdot \sigma_{\mu}-$ $\sigma_{\kappa} \cdot \sigma_{\rho}$ is Schubert positive in $H^{*}\left(G r\left(\ell, \mathbb{C}^{k+\ell}\right), \mathbb{Z}\right)$.

As a special case, consider $\alpha=\left(2^{n+2}\right)$ and $\beta=\left(3,2^{n}, 1\right)$. Setting $\ell=n+2$ and $k=n+3$, so that $N=2 n+4=\ell+k-1$ as required, we have $\lambda=\kappa=$ $(n+1, n, \ldots, 1)=: \delta_{n+1}$, the staircase partition of length $n+1$, while $\mu=(n, n-$ $1, \ldots, 1)=\delta_{n}$ and $v=(n+2, n+1, \ldots, 2)=\delta_{n+2} \backslash 1$. With this notation, the above Theorem 4.3 implies that

$$
\sigma_{\delta_{n+1}} \cdot \sigma_{\delta_{n+1}}-\sigma_{\delta_{n}} \cdot \sigma_{\delta_{n+2} \backslash 1}
$$

is Schubert positive in $H^{*}\left(G r\left(n+2, \mathbb{C}^{2 n+5}\right), \mathbb{Z}\right)$. For example, the $n=2$ case of this implies that

$$
\sigma_{(3,2,1)} \cdot \sigma_{(3,2,1)}-\sigma_{(2,1)} \cdot \sigma_{(4,3,2)}
$$

is Schubert positive in $H^{*}\left(G r\left(4, \mathbb{C}^{9}\right), \mathbb{Z}\right)$.

Although the Schubert positivity of (4.6) can also be established by noting that [8]

$$
s_{\delta_{n+1}} \cdot s_{\delta_{n+1}}-s_{\delta_{n}} \cdot s_{\delta_{n+2} \backslash 1}
$$


is itself Schur positive, this property of Schur functions is not easy to derive.

Moreover, in contrast to this, even though

$$
s_{(4,3,2)} \cdot s_{(3,2,1)}-s_{(5,4,2)} \cdot s_{(3,1)}=-s_{(8,5,2)}+\cdots+s_{(4,3,3,2,2,1)}
$$

is not Schur positive, the corresponding difference of products of Schubert classes

$$
\sigma_{(4,3,2)} \cdot \sigma_{(3,2,1)}-\sigma_{(5,4,2)} \cdot \sigma_{(3,1)}
$$

is Schubert positive in $H^{*}\left(\operatorname{Gr}\left(4, \mathbb{C}^{10}\right), \mathbb{Z}\right)$. This is a consequence of the fact that for $\ell=4$ and $k=6$ we know

$$
s_{(4,3,2)^{c} /(3,2,1)}-s_{(5,4,2)^{c} /(3,1)}=r_{(3,2,2,2)}-r_{(3,3,2,1)}
$$

is Schur positive, by Example 2.14.

Acknowledgements The authors would like to thank Peter McNamara for sharing his conjecture with us and for helpful discussions, and the referees for their constructive comments.

\section{References}

1. Billera, L.J., Thomas, H., van Willigenburg, S.: Decomposable compositions, symmetric quasisymmetric functions and equality of ribbon Schur functions. Adv. Math. 204, 204-240 (2006)

2. Edelman, A., Elmroth, E., Kågström, B.: A geometric approach to perturbation theory of matrices and matrix pencils. Part II: A stratification-enhanced staircase algorithm. SIAM J. Matrix Anal. Appl. 20, 667-699 (1999)

3. Fomin, S., Fulton, W., Li, C.-K., Poon, Y.-T.: Eigenvalues, singular values, and Littlewood-Richardson coefficients. Am. J. Math. 127, 101-127 (2005)

4. Gessel, I.: Multipartite $P$-partitions and inner products of Schur functions. In: Greene, C. (ed.) Combinatorics and Algebra. Contemporary Mathematics, vol. 34. Am. Math. Soc., Providence (1984)

5. Gessel, I., Reutenauer, C.: Counting permutations with given cycle structure and descent set. J. Comb. Theory Ser. A 64, 189-215 (1993)

6. Gutschwager, C.: On multiplicity-free skew characters and the Schubert calculus. Ann. Comb., to appear

7. Hamel, A., Goulden, I.: Planar decompositions of tableaux and Schur function determinants. Eur. J. Comb. 16, 461-477 (1995)

8. Lam, T., Postnikov, A., Pylyavskyy, P.: Schur positivity and Schur log-concavity. Am. J. Math., to appear

9. Macdonald, I.G.: Symmetric Functions and Hall Polynomials, 2nd edn. Oxford University Press, New York (1995)

10. McNamara, P.: Personal communications (2006)

11. Manivel, L.: Symmetric Functions, Schubert Polynomials and Degeneracy loci. AMS Texts and Monographs. Am. Math. Soc., Providence (2001)

12. Okounkov, A.: Log-concavity of multiplicities with application to characters of $U(\infty)$. Adv. Math. 127, 258-282 (1997)

13. Reiner, V., Shaw, K.M., van Willigenburg, S.: Coincidences among skew Schur functions. Adv. Math. 216, 118-152 (2007)

14. Sagan, B.E.: The Symmetric Group: Representations, Combinatorial Algorithms, and Symmetric Functions, 2nd edn. Graduate Texts in Mathematics, vol. 203. Springer, New York (2001)

15. Stanley, R.P.: Enumerative Combinatorics, vol. 2. Cambridge University Press, Cambridge (1999)

16. Thomas, H., Yong, A.: Multiplicity free Schubert calculus. Can. Math. Bull., to appear 